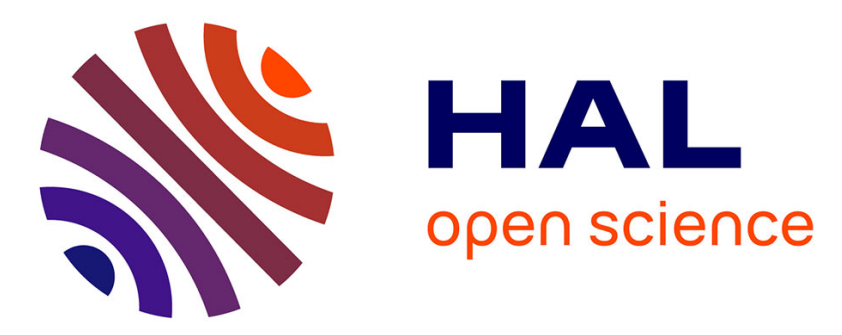

\title{
On the uniqueness and numerical approximations for a matching problem
}

Noureddine Igbida, van Thanh Nguyen, Julian Toledo

\section{To cite this version:}

Noureddine Igbida, van Thanh Nguyen, Julian Toledo. On the uniqueness and numerical approximations for a matching problem. SIAM Journal on Optimization, 2017, 27 (4), pp.2459-2480. hal01549646

\section{HAL Id: hal-01549646 \\ https://hal.science/hal-01549646}

Submitted on 29 Jun 2017

HAL is a multi-disciplinary open access archive for the deposit and dissemination of scientific research documents, whether they are published or not. The documents may come from teaching and research institutions in France or abroad, or from public or private research centers.
L'archive ouverte pluridisciplinaire HAL, est destinée au dépôt et à la diffusion de documents scientifiques de niveau recherche, publiés ou non, émanant des établissements d'enseignement et de recherche français ou étrangers, des laboratoires publics ou privés. 


\title{
ON THE UNIQUENESS AND NUMERICAL APPROXIMATIONS FOR A MATCHING PROBLEM*
}

\author{
NOUREDDINE IGBIDA ${ }^{\dagger}$, VAN THANH NGUYEN ${ }^{\dagger}$, AND JULIÁN TOLEDO $¥$
}

\begin{abstract}
The paper deals with some theoretical and numerical aspects for an optimal matching problem with constraints. It is known that the uniqueness of optimal matching measure does not hold even with $L^{p}$ sources and targets. In this paper, the uniqueness is proven under geometric conditions. On the other hand, we also introduce a dual formulation with a linear cost functional on convex set and show that its Fenchel-Rockafellar dual formulation gives right solution to the optimal matching problem. Basing on our formulations, a numerical approximation is given via an augmented Lagrangian method. We compute at the same time the optimal matching measure, optimal flows and Kantorovich potentials. The convergence of the discretization is also studied in detail.
\end{abstract}

Key words. Optimal matching, optimal transport, Fenchel-Rockafellar duality, augmented Lagrangian methods

AMS subject classifications. 49Q20, 65M60, 65K10, 60K30

1. Introduction. Optimal matching problem (see $[11,9,8]$ and the references therein) deals with the problem to transport two measures of commodities into a prescribed location and to match them there in such a way to minimize the total cost of both transportations. The problem with uniformly convex costs is studied in $[7,11,9,8]$ with applications in economic theory. The case where costs are governed by the Euclidean distance is studied in [19] with connection to $p$-Laplacian type equations.

Optimal constrained matching problem (see [3, 20]), which is a variant from the optimal matching problem and the partial transport problem (see for instance [14, 18]), consists in transporting two kinds of goods and matching them into a target set with constraints on the amount of matter at the target. For example, the target represents the capacities of some companies, the amount of goods matching at each company should have a predetermined bound from above. In mathematical language, the optimal matching problem with constraints for the Euclidean costs can be modeled as follows: Let $\Omega$ be a bounded, convex set of $\mathbb{R}^{N}$ and $f_{1}, f_{2} \in \mathcal{M}_{b}^{+}(\Omega)$ represent source measures of the same mass, i.e., $f_{1}(\Omega)=f_{2}(\Omega)$. The constraint on the target set is represented by a measure $\Theta \in \mathcal{M}_{b}^{+}(\Omega)$, which must satisfy

$$
f_{1}(\Omega)=f_{2}(\Omega)<\Theta(\Omega) .
$$

The optimal matching problem reads

$$
W\left(f_{1}, f_{2} ; \Theta\right):=\inf _{\left(\gamma_{1}, \gamma_{2}\right) \in \pi\left(f_{1}, f_{2} ; \Theta\right)}\left(\int_{\Omega \times \Omega}|x-y| \mathrm{d} \gamma_{1}+\int_{\Omega \times \Omega}|x-y| \mathrm{d} \gamma_{2}\right)
$$

with

$\pi\left(f_{1}, f_{2} ; \Theta\right):=\left\{\left(\gamma_{1}, \gamma_{2}\right) \in \mathcal{M}_{b}^{+}(\Omega \times \Omega)^{2}: \pi_{y} \# \gamma_{1}=\pi_{y} \# \gamma_{2} \leq \Theta, \pi_{x} \# \gamma_{i}=f_{i}, i=1,2\right\}$,

\footnotetext{
* Submitted to the editors November 28, 2016; accepted for publication (in revised form) June 16, 2017.

${ }^{\dagger}$ Institut de recherche XLIM-DMI, UMR-CNRS 6172, Faculté des Sciences et Techniques, Université de Limoges, France (noureddine.igbida@unilim.fr, van-thanh.nguyen@unilim.fr).

‡Departament d'Anàlisi Matemàtica, Universitat de València, Spain (toledojj@uv.es). J. Toledo has been partially supported by the Spanish project MTM2015-70227-P (MINECO/FEDER).
} 
where $\pi_{x} \# \gamma$ and $\pi_{y} \# \gamma$ are the first and second marginals of $\gamma$, respectively. An optimal solution $\left(\gamma_{1}, \gamma_{2}\right)$ is called a couple of optimal plans and $\rho:=\pi_{y} \# \gamma_{1}=\pi_{y} \# \gamma_{2}$ is called an optimal matching measure. Obviously, we can write (1.1) as follows

$$
W\left(f_{1}, f_{2} ; \Theta\right)=\inf _{\rho \in \mathcal{M}_{b}^{+}(\Omega)}\left\{W_{1}\left(f_{1}, \rho\right)+W_{1}\left(f_{2}, \rho\right): \rho \leq \Theta, \rho(\Omega)=f_{1}(\Omega)\right\},
$$

where $W_{1}(.,$.$) is the 1$-Wasserstein distance (see e.g. [21, Chapter 6]). By definition, given two non-negative Radon measures $\mu_{1}$ and $\mu_{2}$ with the same mass on $\Omega$,

$$
W_{1}\left(\mu_{1}, \mu_{2}\right):=\min _{\gamma \in \pi}\left\{\int_{\left(\mu_{1}, \mu_{2}\right)}|x-y| \mathrm{d} \gamma\right\},
$$

where $\pi\left(\mu_{1}, \mu_{2}\right)$ is the set of transport plans, i.e.

$$
\pi\left(\mu_{1}, \mu_{2}\right):=\left\{\gamma \in \mathcal{M}_{b}^{+}(\Omega \times \Omega): \pi_{x} \# \gamma=\mu_{1}, \pi_{y} \# \gamma=\mu_{2}\right\} .
$$

The problem $(\mathrm{OM})$ can be also reformulated by saying that masses moving from $f_{1}$ to $f_{2}$ are forced to pass through an unknown (optimal) distribution less than $\Theta$ and the transportation cost should be optimal. In applications, $f_{1}$ and $f_{2}$ can be distributions of consumers while $\Theta$ would be a distribution of commodities.

Using the direct method in Calculus of Variations, it is not difficult to prove the existence of an optimal matching measure. Our main interest lies in the uniqueness and numerical approximation of the solution. As we will see, the uniqueness of optimal matching measure does not hold even with regular $f_{1}, f_{2}, \Theta$. An additional geometrical condition, as well as the absolute continuity of the measure $\Theta$, is needed for the uniqueness. Concerning the numerical computation, we develop the variational study of the problem.

The optimal constrained matching problem (1.1) is recently studied theoretically in [20] in connection with $p$-Laplacian type systems by using PDE techniques. Inspiring from the work of Evans and Gangbo [13] on the optimal transport theory, the authors in [20] show that an optimal matching measure and associated Kantorovich potentials can be obtained from limits in $p$-Laplacian type equations as $p \rightarrow+\infty$. In [3], Barrett and Prigozhin give a numerical approximation to the problem (1.1) in the case where $\Theta=C \mathcal{L}^{N}\llcorner D$, i.e., $\Theta$ is a constant $C$ on the destination set $D$. Their approach based on nonlinear approximated PDEs. They use Raviart-Thomas finite elements for discretization and solve nonlinear resulting equations.

In this paper, we focus on more variational aspects and the uniqueness of optimal matching measure. We introduce some equivalent formulations for the problem $(\mathrm{OM})$. We give a sufficient condition to ensure the uniqueness of optimal matching measure and show that a solution of the Fenchel-Rockafellar dual formulation is the right solution to the optimal matching problem under a suitable geometric condition. Numerical aspects are also studied with the help of the equivalent formulations. We show the convergence of our approximation and give the details for solving the discretized problems consisting in solving linear equations (with a symmetric positive definite coefficient matrix) or updating an explicit formula.

The paper is organized as follows: In the following section we present our main results such as the uniqueness of optimal matching measure, dual maximization problem, connection between minimal matching flow problem and (OM), the convergence of the discretization and a numerical example illustrating our approach. The details 
and proofs are discussed in the next sections. More precisely, section 3 is devoted to the duality issue while the uniqueness is discussed in section 4. Numerical analysis of the problem is given in section 5 with a study of the convergence of the discretization. We end up the article by giving some numerical examples which validate the approach.

2. Main results. Throughout the paper, $\Omega \subset \mathbb{R}^{N}$ is a bounded convex domain, and $f_{1}, f_{2}, \Theta \in \mathcal{M}_{b}^{+}(\Omega)$ are nonnegative Radon measures such that

$$
f_{1}(\Omega)=f_{2}(\Omega)<\Theta(\Omega) .
$$

It is not difficult to see that the feasible set $\pi\left(f_{1}, f_{2} ; \Theta\right)$ is closed under the weak convergence of Radons measures. This observation gives easily the existence of a couple of optimal plans $\left(\gamma_{1}, \gamma_{2}\right)$ and thus an optimal matching measure $\rho:=\pi_{y} \# \gamma_{1}=$ $\pi_{y} \# \gamma_{2}$. However, in general the uniqueness of optimal matching measures does not hold. For instance, let $f_{1}=\mathcal{L}\left\llcorner[0,1], f_{2}=\mathcal{L}\llcorner[5,6]\right.$ and $\Theta=\mathcal{L}\llcorner[2,4]$, where $\mathcal{L}$ is the Lebesgue measure on $\mathbb{R}$. We see that there are infinitely many optimal matching measures with the total cost $W\left(f_{1}, f_{2} ; \Theta\right)=5$ (one can verify this by using the duality in Theorem 2.3 below).

Here, we prove that under additional conditions on the supports of $\Theta, f_{i}$, for $i=1,2$ and the absolute continuity of $\Theta$, there is a unique optimal matching measure. Let us fix the assumption

$$
S\left(f_{1}, f_{2}\right) \cap \operatorname{supp}(\Theta)=\emptyset
$$

where $S\left(f_{1}, f_{2}\right):=\left\{z=(1-t) x+t y: x \in \operatorname{supp}\left(f_{1}\right), y \in \operatorname{supp}\left(f_{2}\right)\right.$ and $\left.t \in[0,1]\right\}$.

Theorem 2.1. Assume that $\Theta \in L^{1}$ and that $(\mathrm{H})$ holds. There exists a unique optimal matching measure $\rho$.

Notice that the absolute continuity of $\Theta$ is necessary for the uniqueness. Indeed, taking $f_{1}=\delta_{(0,-1)}, f_{2}=\delta_{(0,1)}$ and $\Theta=\delta_{(-1,0)}+\delta_{(1,0)}$ in $\mathbb{R}^{2}$, then $S\left(f_{1}, f_{2}\right) \cap \operatorname{supp}(\Theta)=\emptyset$ and there are again infinitely many optimal matching measures of form $\rho=\alpha \delta_{(-1,0)}+$ $\beta \delta_{(1,0)}$ with $\alpha \geq 0, \beta \geq 0, \alpha+\beta=1$. So, the conditions in Theorem 2.1 are somehow optimal for the uniqueness.

Now, to build a numerical computation of the solution to the optimal matching problem (OM), our main objective is to prove rigorously all the necessary materials to use the augmented Lagrangian method. Our approach is variational. To this aim, we introduce a suitable dual formulation to $(\mathrm{OM})$ which moves the problem into the scope of the general formulation

$$
\inf _{u \in V} \mathcal{F}(u)+\mathcal{G}(\Lambda u)
$$

where $V$ and $Z$ are two Hilbert spaces, $\mathcal{F}: V \longrightarrow(-\infty,+\infty]$ and $\mathcal{G}: Z \longrightarrow(-\infty,+\infty]$ are convex and l.s.c., and $\Lambda \in \mathcal{L}(V, Z)$ the space of continuous linear operators. Once such a dual formulation is given, the ALG2 method (see section 5) can be applied to give numerical solutions to both the problem (2.1) and the Fenchel-Rockafellar dual problem of (2.1):

$$
\sup _{\sigma \in Z^{*}}\left(-\mathcal{F}^{*}\left(-\Lambda^{*} \sigma\right)-\mathcal{G}^{*}(\sigma)\right) .
$$


Recall that the necessary and sufficient condition for optimality of (2.1) and (2.2) reads as

$$
-\Lambda^{*} \sigma \in \partial \mathcal{F}(u) \text { and } \sigma \in \partial \mathcal{G}(\Lambda u)
$$

It is expected that the Fenchel-Rockafellar dual form (2.2) will give informations on the original matching problem. We will see that this is again true under the necessary geometrical condition $(\mathrm{H})$.

We come back to the duality issue for $(\mathrm{OM})$. As usual, let us denote by $\operatorname{Lip}_{1}(\Omega)$ the set of 1-Lipschitz functions on $\Omega$. By extension, we usually identify $\operatorname{Lip}_{1}(\Omega)$ with $\operatorname{Lip}_{1}(\bar{\Omega})$. Let us recall that the duality issue was already studied in [20] with the following result:

Theorem $2.2([20])$. Assume that $f_{1}, f_{2} \in L^{\infty}(\Omega)$. One has

$$
W\left(f_{1}, f_{2} ; \Theta\right)=\max \left\{-\int u_{1} \mathrm{~d} f_{1}-\int u_{2} \mathrm{~d} f_{2}-\int\left(u_{1}+u_{2}\right)^{-} \mathrm{d} \Theta: u_{1}, u_{2} \in \operatorname{Lip}_{1}(\Omega)\right\} .
$$

However, even if the maximization problem in (2.4) falls into the scope of (2.1), unfortunately the corresponding $\mathcal{F}$ is nonlinear on its variable $u:=\left(u_{1}, u_{2}\right)$ and $(2.4)$ is not very useful for the numerical computation. Here, we introduce a new dual formulation with the following linear cost functional:

$$
\max \left\{\int\left(u_{1}+u_{2}\right) \mathrm{d} \Theta-\int u_{1} \mathrm{~d} f_{1}-\int u_{2} \mathrm{~d} f_{2}:\left(u_{1}, u_{2}\right) \in K\right\},
$$

where

$$
K:=\left\{\left(u_{1}, u_{2}\right) \in \operatorname{Lip}_{1}(\Omega) \times \operatorname{Lip}_{1}(\Omega): u_{1}+u_{2} \leq 0\right\} .
$$

Using the Fenchel-Rockafellar dual theory for the maximization problem (2.5), we also introduce the minimal matching flow (MMF) problem:

$$
\min \left\{\left|\Phi_{1}\right|(\bar{\Omega})+\left|\Phi_{2}\right|(\bar{\Omega}):\left(\Phi_{1}, \Phi_{2}, \nu\right) \in \Psi\left(f_{1}, f_{2} ; \Theta\right)\right\}
$$

where

$$
\begin{aligned}
& \Psi\left(f_{1}, f_{2} ; \Theta\right) \\
& :=\left\{\left(\Phi_{1}, \Phi_{2}, \nu\right) \in \mathcal{M}_{b}(\bar{\Omega})^{N} \times \mathcal{M}_{b}(\bar{\Omega})^{N} \times \mathcal{M}_{b}^{+}(\bar{\Omega}):-\nabla \cdot \Phi_{i}=\Theta-\nu-f_{i} \text { in } \mathbb{R}^{N}, i=1,2\right\} .
\end{aligned}
$$

Here, the divergence constraint is understood in the sense of distribution, i.e.,

$$
\left\langle\nabla \phi, \Phi_{i}\right\rangle_{\left(C(\bar{\Omega})^{N}, \mathcal{M}_{b}(\bar{\Omega})^{N}\right)}=\int_{\bar{\Omega}} \nabla \phi \cdot \frac{\Phi_{i}}{\left|\Phi_{i}\right|} \mathrm{d}\left|\Phi_{i}\right|=\int_{\bar{\Omega}} \phi \mathrm{d}\left(\Theta-\nu-f_{i}\right),
$$

for any smooth compactly supported function $\phi \in C_{c}^{\infty}\left(\mathbb{R}^{N}\right)$. We denote by $\frac{\Phi}{|\Phi|}$ the density of $\Phi$ w.r.t. the measure $|\Phi|$ and by $\nabla_{|\Phi|} u$ the tangential gradient w.r.t. $|\Phi|$, which is well-defined for any Lipschitz function $u$ (see e.g. [6]).

Our main result concerning duality and quivalent formulations is summarized in the following theorem.

Theorem 2.3. Let $f_{1}, f_{2}, \Theta$ be Radon measures. We have

$$
\begin{aligned}
W\left(f_{1}, f_{2} ; \Theta\right) & =\max \left\{\int\left(u_{1}+u_{2}\right) \mathrm{d} \Theta-\int u_{1} \mathrm{~d} f_{1}-\int u_{2} \mathrm{~d} f_{2}:\left(u_{1}, u_{2}\right) \in K\right\} \\
& =\min \left\{\left|\Phi_{1}\right|(\bar{\Omega})+\left|\Phi_{2}\right|(\bar{\Omega}):\left(\Phi_{1}, \Phi_{2}, \nu\right) \in \Psi\left(f_{1}, f_{2} ; \Theta\right)\right\} .
\end{aligned}
$$


Moreover, we have that

- $\left(\gamma_{1}, \gamma_{2}\right) \in \pi\left(f_{1}, f_{2} ; \Theta\right)$ and $\left(u_{1}, u_{2}\right) \in K$ are optimal for the optimal constrained matching problem (1.1) and the maximization problem (2.5), respectively, if and only if

$$
\left\{\begin{array}{l}
u_{1}+u_{2}=0,(\Theta-\rho) \text {-a.e., with } \rho:=\pi_{y} \# \gamma_{1}=\pi_{y} \# \gamma_{2} \\
u_{1}(y)-u_{1}(x)=|y-x| \text { for all }(x, y) \in \operatorname{supp}\left(\gamma_{1}\right) \\
u_{2}(y)-u_{2}(x)=|y-x| \text { for all }(x, y) \in \operatorname{supp}\left(\gamma_{2}\right) .
\end{array}\right.
$$

- $\left(u_{1}, u_{2}\right) \in K$ and $\left(\Phi_{1}, \Phi_{2}, \nu\right) \in \Psi\left(f_{1}, f_{2} ; \Theta\right)$ are optimal for (2.5) and (MMF), respectively, if and only if the following optimality condition holds

$$
\left\{\begin{array}{l}
-\nabla \cdot \Phi_{i}=\Theta-\nu-f_{i} \text { in } \mathbb{R}^{N}, i=1,2 \\
\frac{\Phi_{i}}{\left|\Phi_{i}\right|}=\nabla_{\left|\Phi_{i}\right|} u_{i}\left|\Phi_{i}\right| \text {-a.e. in } \bar{\Omega}, i=1,2 \\
u_{1}+u_{2}=0 \quad \nu \text {-a.e. in } \bar{\Omega} .
\end{array}\right.
$$

Remark 2.4. If $\Theta$ is absolutely continuous, the optimality condition (2.8) can be simplified by using the usual gradient instead of the tangential gradient. In fact, in this case, it is known that $\Phi_{i}$ is also absolutely continuous (see for instance [1]) and that $u$ is then differentiable $\left|\Phi_{i}\right|$-a.e.. By regularization via convolution, we can use $u$ as test function in the first equation of (2.8), and using the duality (2.6), we get $\frac{\Phi_{i}}{\left|\Phi_{i}\right|}=\nabla u_{i}$ for $\left|\Phi_{i}\right|$-a.e. in $\bar{\Omega}$.

Roughly speaking, the dual maximization formulation (2.5), the problem (MMF) and the system (2.8) correspond to (2.1), (2.2) and the optimality condition (2.3), respectively. In the optimal mass transportation problem, these three formulations contain all the informations concerning the optimal transportation. This is extensively used to give numerical approximations for some variants of the optimal mass transport problem (see for instance $[4,5,17]$ ). For the optimal matching problem, we need to compute moreover the optimal matching measure. As an immediate consequence of the duality equalities in Theorem 2.3, the following result shows how this can be carried out.

CoROLlary 2.5. Let $\rho$ be an optimal matching measure and $\Phi_{i}$ be optimal flows for transporting $f_{i}$ onto $\rho, i=1,2$. Then $\left(\Phi_{1}, \Phi_{2}, \nu\right):=\left(\Phi_{1}, \Phi_{2}, \Theta-\rho\right)$ is an optimal solution for the associated problem (MMF). Conversely, if $\left(\Phi_{1}, \Phi_{2}, \nu\right)$ is an optimal solution for the problem (MMF) and $\nu \leq \Theta$, then $\rho:=\Theta-\nu$ is an optimal matching measure and $\Phi_{i}$ is an optimal flow of transporting $f_{i}$ onto $\rho, i=1,2$.

This result shows that the connection between (MMF) and (OM) lies in the condition $\nu \leq \Theta$ for an optimal solution $\left(\Phi_{1}, \Phi_{2}, \nu\right)$ of (MMF). Unfortunately, this does not hold in general as shown in the following example.

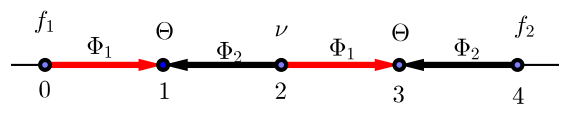

Fig. 1: Example of $\nu \nless \Theta$

Example 2.6. On $\mathbb{R}$, taking $f_{1}=\delta_{0}, f_{2}=\delta_{4}, \Theta=\delta_{1}+\delta_{3}$, where $\delta_{i}$ is the Dirac mass at $i$ on $\mathbb{R}$ (see Figure 1). Let $\nu=\delta_{2}$ and $\Phi_{1}$ be the optimal flow of transporting 
$f_{1}+\nu$ onto $\Theta$ (the corresponding plan is described as follows: $f_{1}=\delta_{0} \rightarrow \delta_{1}, \nu=\delta_{2} \rightarrow$ $\delta_{3}$ ) and $\Phi_{2}$ be the optimal flow of transporting $f_{2}+\nu$ onto $\Theta$ (the corresponding plan is described as follows: $\left.f_{2}=\delta_{4} \rightarrow \delta_{3}, \nu=\delta_{2} \rightarrow \delta_{1}\right)$. Then $\left(\Phi_{1}, \Phi_{2}, \nu\right)$ is an optimal solution of the problem (MMF). Indeed, it is not difficult to see that the total cost of matching $f_{1}$ and $f_{2}$ into $\Theta$ is 4 . The cost of the problem (MMF) corresponding to this choice of $\left(\Phi_{1}, \Phi_{2}, \nu\right)$ is also 4 . From our duality results, we have the optimality of $\left(\Phi_{1}, \Phi_{2}, \nu\right)$, but $\nu \nless \Theta$.

However, under the assumption $(\mathrm{H})$, we prove that the constraint $\nu \leq \Theta$ is fulfilled. More precisely, we have

Theorem 2.7. Let $f_{1}, f_{2}, \Theta \in \mathcal{M}_{b}^{+}(\Omega)$ be Radon measures. Assume that $(\mathrm{H})$ holds. Let $\left(\Phi_{1}, \Phi_{2}, \nu\right) \in \Psi\left(f_{1}, f_{2} ; \Theta\right)$ be an optimal solution for the problem (MMF) and set $\rho:=\Theta-\nu$. Then $\rho \geq 0$ and it is an optimal matching measure.

Before ending up this section let us show how we use the ALG2 method to solve numerically the optimal matching problem $(\mathrm{OM})$. For any $u=\left(u_{1}, u_{2}\right) \in V:=$ $C^{1}(\bar{\Omega}) \times C^{1}(\bar{\Omega})$, we set

$$
\begin{gathered}
\mathcal{F}(u):=\int u_{1} \mathrm{~d} f_{1}+\int u_{2} \mathrm{~d} f_{2}-\int\left(u_{1}+u_{2}\right) \mathrm{d} \Theta \\
\Lambda(u):=\left(\nabla u_{1}, \nabla u_{2}, u_{1}+u_{2}\right),
\end{gathered}
$$

and, for any $(p, q, s) \in Z:=C(\bar{\Omega})^{N} \times C(\bar{\Omega})^{N} \times C(\bar{\Omega})$, we set

$$
\mathcal{G}(p, q, s):= \begin{cases}0 & \text { if }|p(x)| \leq 1,|q(x)| \leq 1, s(x) \leq 0 \quad \forall x \in \bar{\Omega} \\ +\infty & \text { otherwise. }\end{cases}
$$

Then the problem

$$
\inf _{u \in V} \mathcal{F}(u)+\mathcal{G}(\Lambda u)
$$

provides all information on the optimal matching problem. Indeed, $u_{1}, u_{2}$ give Kantorovich potentials and dual variables $\Phi_{1}, \Phi_{2}, \nu$ give information on optimal flows and optimal matching measure. To solve numerically the problem (2.9) and its FenchelRockafellar dual formulation (MMF), we consider a regular triangulation $\mathcal{T}_{h}$ of $\bar{\Omega}$. We fixe an integer $k \geq 1$, and we consider $P_{k}$ the set of polynomials of degree less or equal than $k$. Let $E_{h} \subset H^{1}(\Omega)$ be the space of continuous functions on $\bar{\Omega}$ and belonging to $P_{k}$ on each triangle of $\mathcal{T}_{h}$. We denote by $Y_{h}$ the space of vectorial functions such that their restrictions belong to $\left(P_{k-1}\right)^{N}$ on each triangle of $\mathcal{T}_{h}$. Let $f_{1, h}, f_{2, h}, \Theta_{h} \in E_{h}$ such that $f_{1, h}(\Omega)=f_{2, h}(\Omega)<\Theta_{h}(\Omega)$ and $f_{1, h} \rightarrow f_{1}, f_{2, h} \rightarrow f_{2}, \Theta_{h} \rightarrow \Theta$ weakly* in $\mathcal{M}_{b}(\bar{\Omega})$. Set $V_{h}:=E_{h} \times E_{h}$ and $Z_{h}:=Y_{h} \times Y_{h} \times E_{h}$. We approximate the problem (2.9) by the following finite-dimensional problem: For any $\left(u_{1}, u_{2}\right) \in V_{h}$, we set

$$
\begin{gathered}
\Lambda_{h}\left(u_{1}, u_{2}\right):=\left(\nabla u_{1}, \nabla u_{2}, u_{1}+u_{2}\right) \in Z_{h}, \\
\mathcal{F}_{h}\left(u_{1}, u_{2}\right):=\left\langle u_{1}, f_{1, h}\right\rangle+\left\langle u_{2}, f_{2, h}\right\rangle-\left\langle u_{1}+u_{2}, \Theta_{h}\right\rangle,
\end{gathered}
$$

and for any $(p, q, s) \in Z_{h}$,

$$
\mathcal{G}_{h}(p, q, s):= \begin{cases}0 & \text { if }|p(x)| \leq 1,|q(x)| \leq 1, s(x) \leq 0 \text { a.e. } x \in \Omega \\ +\infty & \text { otherwise }\end{cases}
$$


The finite-dimensional approximation of (2.9) is given by

$$
\inf _{\left(u_{1}, u_{2}\right) \in V_{h}} \mathcal{F}_{h}\left(u_{1}, u_{2}\right)+\mathcal{G}_{h}\left(\Lambda_{h}\left(u_{1}, u_{2}\right)\right)
$$

Note that the cost functional does not change under the translation $\tilde{u}_{1}:=u_{1}+C, \tilde{u}_{2}:=$ $u_{2}-C$, for $C \in \mathbb{R}$. In particular, the new couple

$$
\left(\tilde{u}_{1}:=u_{1}-\frac{|\Omega|}{2} \int_{\Omega}\left(u_{1}-u_{2}\right), \tilde{u}_{2}:=u_{2}+\frac{|\Omega|}{2} \int_{\Omega}\left(u_{1}-u_{2}\right)\right)
$$

satisfies $\int_{\Omega} \tilde{u}_{1}=\int_{\Omega} \tilde{u}_{2}$ and is optimal if $\left(u_{1}, u_{2}\right)$ is optimal.

The next theorem shows that (2.10) is a suitable approximation of (2.9) in the sense that primal and dual solutions converge to a solution of (2.9) (i.e. a solution of the maximization problem (2.5)) and a solution of (MMF).

THEOREM 2.8. Let $\left(u_{1, h}, u_{2, h}\right) \in V_{h}$ be an optimal solution to the approximated problem (2.10) such that $\int_{\Omega} u_{1, h}=\int_{\Omega} u_{2, h}$ and let $\left(\Phi_{1, h}, \Phi_{2, h}, \nu_{h}\right)$ be an optimal dual solution to (2.10). Then, up to a subsequence, $\left(u_{1, h}, u_{2, h}\right)$ converges uniformly to $\left(u_{1}^{*}, u_{2}^{*}\right)$ an optimal solution of the dual maximization problem (2.5) and $\left(\Phi_{1, h}, \Phi_{2, h}, \nu_{h}\right)$ converges weakly* to $\left(\Phi_{1}, \Phi_{2}, \nu\right)$ an optimal solution of (MMF).

At last, we solve the finite-dimensional problem (2.10) by using the ALG2 method. The details of the method are given in section 5. Here, we just give an illustration of our numerical results on the following example (see Figure 2): In $\mathbb{R}^{2}$, we take $\Omega=[0,1] \times[0,1]$

$$
f_{1}=4 \chi_{\left[(x-0.2)^{2}+(y-0.8)^{2}<0.01\right]}, f_{2}=4 \chi_{\left[(x-0.2)^{2}+(y-0.2)^{2}<0.01\right]},
$$

and

$$
\Theta=4 \chi_{\left[(x-0.8)^{2}+(y-0.2)^{2}<0.04\right]},
$$

where $\chi_{A}(x)$ is the characteristic function of $A$.

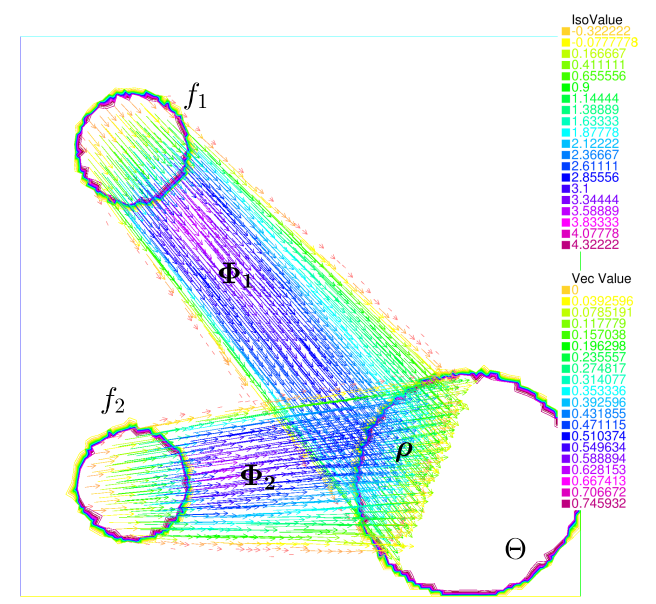

Fig. 2: Optimal matching measure $\rho$ and optimal flows $\Phi_{1}$ and $\Phi_{2}$ 
3. Proofs of the equivalent formulations. The present section deals with the proofs of dual formulations as well as the connection between the minimal matching flow and the optimal constrained matching problem. To begin with, let us recall some well-known results in optimal transport theory with the Euclidean cost. It is well-known that the optimal transport problem (1.2) has the dual formulation

$$
\max \left\{\int_{\Omega} u \mathrm{~d}\left(\mu_{2}-\mu_{1}\right): u \in \operatorname{Lip}_{1}(\Omega)\right\}
$$

i.e.,

$$
W_{1}\left(\mu_{1}, \mu_{2}\right)=\min _{\gamma \in \pi_{\left(\mu_{1}, \mu_{2}\right)}}\left\{\int_{\Omega \times \Omega}|x-y| \mathrm{d} \gamma\right\}=\max _{u \in L i p_{1}(\Omega)}\left\{\int_{\Omega} u \mathrm{~d}\left(\mu_{2}-\mu_{1}\right)\right\} .
$$

It follows immediately from (3.2) that the 1-Wasserstein distance depends only on the difference of its arguments. On the other hand, the Fenchel-Rockafellar dual formulation of (3.1), called minimal flow problem, reads as

$$
W_{1}\left(\mu_{1}, \mu_{2}\right)=\min _{\Phi \in \mathcal{M}_{b}(\bar{\Omega})^{N}}\left\{|\Phi|(\bar{\Omega}):-\nabla \cdot \Phi=\mu_{2}-\mu_{1} \text { in } \mathbb{R}^{N}\right\} .
$$

Optimizers of (3.1) and (3.3) are called Kantorovich potentials and optimal flows, respectively. These optimizers are completely characterized by the following PDE

$$
\left\{\begin{array}{l}
-\nabla \cdot \Phi=\mu_{2}-\mu_{1} \text { in } \mathbb{R}^{N} \\
\frac{\Phi}{|\Phi|}=\nabla_{|\Phi|} u \quad|\Phi| \text {-a.e. in } \bar{\Omega} \\
u \in \operatorname{Lip}_{1}(\Omega) .
\end{array}\right.
$$

This fact can be done by using the integration by parts formula (see for instance [6]):

$$
\forall \psi \in \operatorname{Lip}_{1}(\Omega), \quad\langle\nabla \cdot \Phi, \psi\rangle=\int \nabla_{|\Phi|} \psi \frac{\Phi}{|\Phi|} \mathrm{d}|\Phi| .
$$

Coming back to the optimal constrained matching problem, we start with the Fenchel-Rockafellar duality between (2.5) and (MMF).

Lemma 3.1. Let $f_{1}, f_{2}, \Theta \in \mathcal{M}_{b}^{+}(\Omega)$ be Radon measures. We have

$$
\begin{aligned}
& \max \left\{\int\left(u_{1}+u_{2}\right) \mathrm{d} \Theta-\int u_{1} \mathrm{~d} f_{1}-\int u_{2} \mathrm{~d} f_{2}:\left(u_{1}, u_{2}\right) \in K\right\} \\
& =\min \left\{\left|\Phi_{1}\right|(\bar{\Omega})+\left|\Phi_{2}\right|(\bar{\Omega}):\left(\Phi_{1}, \Phi_{2}, \nu\right) \in \Psi\left(f_{1}, f_{2} ; \Theta\right)\right\} .
\end{aligned}
$$

Keeping in mind the use of augmented Lagrangian method for numerical approximations, we use the Fenchel-Rockafellar duality technique to prove Lemma 3.1. tion,

Proof. We observe that, using the standard smooth approximation by convolu-

$$
\begin{aligned}
& \max \left\{\int\left(u_{1}+u_{2}\right) \mathrm{d} \Theta-\int u_{1} \mathrm{~d} f_{1}-\int u_{2} \mathrm{~d} f_{2}:\left(u_{1}, u_{2}\right) \in K\right\} \\
& =\sup \left\{\int\left(u_{1}+u_{2}\right) \mathrm{d} \Theta-\int u_{1} \mathrm{~d} f_{1}-\int u_{2} \mathrm{~d} f_{2}:\left(u_{1}, u_{2}\right) \in K, u_{1}, u_{2} \in C^{1}(\bar{\Omega})\right\} \\
& =-\inf _{\left(u_{1}, u_{2}\right) \in C^{1}(\bar{\Omega}) \times C^{1}(\bar{\Omega})} \mathcal{F}\left(u_{1}, u_{2}\right)+\mathcal{G}\left(\Lambda\left(u_{1}, u_{2}\right)\right),
\end{aligned}
$$


where $\mathcal{F}, \mathcal{G}$ and $\Lambda$ are given in section 2. Now, using the Fenchel-Rockafellar dual theory (see for instance [12, Chapter III, Remark 4.2]), we have

$$
\begin{gathered}
\inf _{\left(u_{1}, u_{2}\right) \in C^{1}(\bar{\Omega}) \times C^{1}(\bar{\Omega})} \mathcal{F}\left(u_{1}, u_{2}\right)+\mathcal{G}\left(\Lambda\left(u_{1}, u_{2}\right)\right) \\
=\max _{\left(\Phi_{1}, \Phi_{2}, \nu\right) \in \mathcal{M}_{b}(\bar{\Omega})^{N} \times \mathcal{M}_{b}(\bar{\Omega})^{N} \times \mathcal{M}_{b}(\bar{\Omega})}\left\{-\mathcal{F}^{*}\left(-\Lambda^{*}\left(\Phi_{1}, \Phi_{2}, \nu\right)\right)-\mathcal{G}^{*}\left(\Phi_{1}, \Phi_{2}, \nu\right)\right\} .
\end{gathered}
$$

So, it is enough to compute explicitly the above quantities. Since $\mathcal{F}$ is linear, the quantity $\mathcal{F}^{*}\left(-\Lambda^{*}\left(\Phi_{1}, \Phi_{2}, \nu\right)\right)$ is finite (and is thus equal to 0 ) if and only if

$$
\left\langle-\Lambda^{*}\left(\Phi_{1}, \Phi_{2}, \nu\right),\left(u_{1}, u_{2}\right)\right\rangle=\mathcal{F}\left(u_{1}, u_{2}\right)=\int u_{1} \mathrm{~d} f_{1}+\int u_{2} \mathrm{~d} f_{2}-\int\left(u_{1}+u_{2}\right) \mathrm{d} \Theta
$$

for all $\left(u_{1}, u_{2}\right) \in C^{1}(\bar{\Omega}) \times C^{1}(\bar{\Omega})$, or

$$
-\left\langle\Phi_{1}, \nabla u_{1}\right\rangle-\left\langle\Phi_{2}, \nabla u_{2}\right\rangle-\left\langle\nu, u_{1}+u_{2}\right\rangle=\int u_{1} \mathrm{~d} f_{1}+\int u_{2} \mathrm{~d} f_{2}-\int\left(u_{1}+u_{2}\right) \mathrm{d} \Theta
$$

for all $\left(u_{1}, u_{2}\right) \in C^{1}(\bar{\Omega}) \times C^{1}(\bar{\Omega})$. This implies that (by taking $\left(u_{1}, u_{2}\right)=\left(u_{1}, 0\right)$ and $\left(u_{1}, u_{2}\right)=\left(0, u_{2}\right)$ as test functions $)$

$$
-\nabla \cdot \Phi_{i}=\Theta-\nu-f_{i} \text { in } \mathbb{R}^{N}, i=1,2 .
$$

Next, it is easy to see that

$$
\mathcal{G}^{*}\left(\Phi_{1}, \Phi_{2}, \nu\right)= \begin{cases}\left|\Phi_{1}\right|(\bar{\Omega})+\left|\Phi_{2}\right|(\bar{\Omega}) & \text { if } \nu \geq 0, \\ +\infty, & \text { otherwise }\end{cases}
$$

Therefore the proof is completed by substituting $\mathcal{F}^{*}$ and $\mathcal{G}^{*}$ into (3.6).

Following immediately from (3.3), we see that

$$
\begin{aligned}
& \min \left\{\left|\Phi_{1}\right|(\bar{\Omega})+\left|\Phi_{2}\right|(\bar{\Omega}):\left(\Phi_{1}, \Phi_{2}, \nu\right) \in \Psi\left(f_{1}, f_{2} ; \Theta\right)\right\} \\
& =\min _{\nu \in \mathcal{M}_{b}^{+}(\bar{\Omega})}\left\{W_{1}\left(f_{1}+\nu, \Theta\right)+W_{1}\left(f_{2}+\nu, \Theta\right): \nu(\bar{\Omega})=\Theta(\Omega)-f_{1}(\Omega)\right\} .
\end{aligned}
$$

This proposes an alternative formulation of $(\mathrm{OM})$ that we prove directly in the following lemma.

Lemma 3.2. Assume that $f_{1}, f_{2}, \Theta \in \mathcal{M}_{b}^{+}(\Omega)$ are Radon measures. We have

$$
\begin{aligned}
& \min _{\rho \in \mathcal{M}_{b}^{+}(\Omega)}\left\{W_{1}\left(f_{1}, \rho\right)+W_{1}\left(f_{2}, \rho\right): \rho \leq \Theta, \rho(\Omega)=f_{1}(\Omega)\right\} \\
= & \min _{\nu \in \mathcal{M}_{b}^{+}(\bar{\Omega})}\left\{W_{1}\left(f_{1}+\nu, \Theta\right)+W_{1}\left(f_{2}+\nu, \Theta\right): \nu(\bar{\Omega})=\Theta(\Omega)-f_{1}(\Omega)\right\} .
\end{aligned}
$$

Moreover, if $\nu$ is optimal for the right hand side of (3.8) then there exist $0 \leq \theta_{1}, \theta_{2} \leq$ $\Theta, \theta_{1}(\Omega)=\theta_{2}(\Omega)=f_{1}(\Omega)$ such that

$$
\begin{aligned}
& W_{1}\left(f_{1}, \theta_{2}\right)=W_{1}\left(f_{1}, \theta_{1}\right)+W_{1}\left(\theta_{1}, \theta_{2}\right), \\
& W_{1}\left(f_{2}, \theta_{1}\right)=W_{1}\left(f_{2}, \theta_{2}\right)+W_{1}\left(\theta_{1}, \theta_{2}\right)
\end{aligned}
$$

and

$$
W_{1}\left(\nu, \Theta-\theta_{1}\right)+W_{1}\left(\nu, \Theta-\theta_{2}\right)=W_{1}\left(\theta_{1}, \theta_{2}\right)
$$


Proof. The existence of minimizers follows easily from the direct method. Now, fix any $\rho \in \mathcal{M}_{b}^{+}(\Omega)$ with $\rho \leq \Theta, \rho(\Omega)=f_{1}(\Omega)$ and set $\nu:=\Theta-\rho$. We have

$$
W_{1}\left(f_{1}, \rho\right)+W_{1}\left(f_{2}, \rho\right)=W_{1}\left(f_{1}+\nu, \Theta\right)+W_{1}\left(f_{2}+\nu, \Theta\right) .
$$

This shows that the left hand side of (3.8) is greater than or equal to the right hand side. Conversely, take $\nu \in \mathcal{M}_{b}^{+}(\bar{\Omega})$ with $\nu(\bar{\Omega})=\Theta(\Omega)-f_{1}(\Omega)$. Consider the optimal plan $\gamma_{i}$ between $f_{i}+\nu$ and $\Theta$. It sends $f_{i}$ to some $\theta_{i} \leq \Theta, i=1,2$ such that

$W_{1}\left(f_{1}+\nu, \Theta\right)=W_{1}\left(f_{1}, \theta_{1}\right)+W_{1}\left(\nu, \Theta-\theta_{1}\right), W_{1}\left(f_{2}+\nu, \Theta\right)=W_{1}\left(f_{2}, \theta_{2}\right)+W_{1}\left(\nu, \Theta-\theta_{2}\right)$,

$$
f_{1}(\Omega)=\theta_{1}(\Omega)=\theta_{2}(\Omega) .
$$

By triangular inequality and $W_{1}\left(\Theta-\theta_{1}, \Theta-\theta_{2}\right)=W_{1}\left(\theta_{1}, \theta_{2}\right)$, we get

$(3.12)$

$$
\begin{aligned}
W_{1}\left(f_{1}+\nu, \Theta\right)+W_{1}\left(f_{2}+\nu, \Theta\right) & =W_{1}\left(f_{1}, \theta_{1}\right)+W_{1}\left(\nu, \Theta-\theta_{1}\right)+W_{1}\left(f_{2}, \theta_{2}\right)+W_{1}\left(\nu, \Theta-\theta_{2}\right) \\
& \geq W_{1}\left(f_{1}, \theta_{1}\right)+W_{1}\left(f_{2}, \theta_{2}\right)+W_{1}\left(\theta_{1}, \theta_{2}\right) \\
& \geq \max _{i=1,2}\left\{W_{1}\left(f_{1}, \theta_{i}\right)+W_{1}\left(f_{2}, \theta_{i}\right)\right\} \\
& \geq \min _{i=1,2}\left\{W_{1}\left(f_{1}, \theta_{i}\right)+W_{1}\left(f_{2}, \theta_{i}\right)\right\} \\
& \geq \min _{\rho \in \mathcal{M}_{b}^{+}(\Omega)}\left\{W_{1}\left(f_{1}, \rho\right)+W_{1}\left(f_{2}, \rho\right): \rho \leq \Theta, \rho(\Omega)=f_{1}(\Omega)\right\} .
\end{aligned}
$$

Therefore, the proof of the equality (3.8) is done.

At last, if $\nu$ is optimal then all the inequalities in (3.12) become equalities. This implies the conclusions (3.9), (3.10) and (3.11).

Proof of Theorem 2.3. The duality (2.6) follows from (3.7), Lemmas 3.1 and 3.2. It remains to show the optimality conditions (2.7) and (2.8). Let us begin with the proof of (2.7). For any admissible $\left(u_{1}, u_{2}\right) \in K$ and $\left(\gamma_{1}, \gamma_{2}\right) \in \pi\left(f_{1}, f_{2} ; \Theta\right)$, taking $\rho:=\pi_{y} \# \gamma_{1}=\pi_{y} \# \gamma_{2}$, we have

$$
\begin{aligned}
& \int\left(u_{1}+u_{2}\right) \mathrm{d} \Theta-\int u_{1} \mathrm{~d} f_{1}-\int u_{2} \mathrm{~d} f_{2} \\
& \leq \int\left(u_{1}+u_{2}\right) \mathrm{d} \rho-\int u_{1} \mathrm{~d} f_{1}-\int u_{2} \mathrm{~d} f_{2} \\
& =\int\left(u_{1}(y)-u_{1}(x)\right) \mathrm{d} \gamma_{1}+\int\left(u_{2}(y)-u_{2}(x)\right) \mathrm{d} \gamma_{2} \\
& \leq \int|x-y| \mathrm{d} \gamma_{1}+\int|x-y| \mathrm{d} \gamma_{2} .
\end{aligned}
$$

From the duality equalities (2.6), we deduce that $\left(\gamma_{1}, \gamma_{2}\right)$ and $\left(u_{1}, u_{2}\right)$ are optimal if and only if all the inequalities in (3.13) become equalities. The latter conditions read as

$$
\left\{\begin{array}{l}
\int\left(u_{1}+u_{2}\right) \mathrm{d} \Theta=\int\left(u_{1}+u_{2}\right) \mathrm{d} \rho \\
\int\left(u_{1}(y)-u_{1}(x)\right) \mathrm{d} \gamma_{1}=\int|x-y| \mathrm{d} \gamma_{1} \\
\int\left(u_{2}(y)-u_{2}(x)\right) \mathrm{d} \gamma_{2}=\int|x-y| \mathrm{d} \gamma_{2} .
\end{array}\right.
$$

This condition is equivalent to (2.7).

For the proof of $(2.8)$, we see that, for any admissible $\left(\Phi_{1}, \Phi_{2}, \nu\right) \in \Psi\left(f_{1}, f_{2} ; \Theta\right)$, by 
the integration by parts formula (3.5), we have (3.14)

$$
\begin{aligned}
-\int u_{1} \mathrm{~d} f_{1}-\int u_{2} \mathrm{~d} f_{2}+\int\left(u_{1}+u_{2}\right) \mathrm{d} \Theta & \leq-\int u_{1} \mathrm{~d} f_{1}-\int u_{2} \mathrm{~d} f_{2}+\int\left(u_{1}+u_{2}\right) \mathrm{d}(\Theta-\nu) \\
& =\int u_{1} \mathrm{~d}\left(\Theta-\nu-f_{1}\right)+\int u_{2} \mathrm{~d}\left(\Theta-\nu-f_{2}\right) \\
& =\int_{\bar{\Omega}} \frac{\Phi_{1}}{\left|\Phi_{1}\right|} \cdot \nabla_{\left|\Phi_{1}\right|} u_{1} \mathrm{~d}\left|\Phi_{1}\right|+\int_{\bar{\Omega}} \frac{\Phi_{2}}{\left|\Phi_{2}\right|} \cdot \nabla_{\left|\Phi_{2}\right|} u_{2} \mathrm{~d}\left|\Phi_{2}\right| \\
& \leq\left|\Phi_{1}\right|(\bar{\Omega})+\left|\Phi_{2}\right|(\bar{\Omega}) .
\end{aligned}
$$

Thanks to (2.6), $\left(u_{1}, u_{2}\right)$ and $\left(\Phi_{1}, \Phi_{2}, \nu\right)$ are optimal if and only if all the inequalities in (3.14) become equalities. This is equivalent to the system (2.8).

We end up this section by giving the proof of Theorem 2.7 concerning the relation between (MMF) and (OM).

Proof of Theorem 2.7. Assume that $\left(\Phi_{1}, \Phi_{2}, \nu\right)$ is optimal for (MMF) which implies that $\nu$ is optimal for the alternative formulation of $(\mathrm{OM})$ given in Lemma 3.2. Take $\theta_{1}$ and $\theta_{2}$ given by Lemma 3.2. Then (3.9) and (3.10) mean that $\theta_{2}$ is on a geodesic joining $\theta_{1}$ to $f_{2}$ and $\theta_{1}$ is on a geodesic joining $\theta_{2}$ to $f_{1}$. The assumption $(\mathrm{H})$ imposes that $\theta_{1}=\theta_{2}$. To convince the reader, take $\gamma_{f_{1}, 1}, \gamma_{1,2}$ and $\gamma_{2, f_{2}}$ the optimal plans from $f_{1}$ to $\theta_{1}$, from $\theta_{1}$ to $\theta_{2}$ and from $\theta_{2}$ to $f_{2}$. Using the gluing lemma (see e.g. [21, Lemma 7.6]), we build $\gamma_{f_{1}, 1,2}$ obtained by gluing $\gamma_{f_{1}, 1}$ to $\gamma_{1,2}$ and $\gamma_{1,2, f_{2}}$ obtained by gluing $\gamma_{1,2}$ to $\gamma_{2, f_{2}}$. Then, it holds

$$
\begin{gathered}
W_{1}\left(f_{1}, \theta_{1}\right)+W_{1}\left(\theta_{1}, \theta_{2}\right)=\int_{\bar{\Omega}^{3}}\left|x_{1}-z_{1}\right|+\left|z_{1}-z_{2}\right| \mathrm{d} \gamma_{f_{1}, 1,2}\left(x_{1}, z_{1}, z_{2}\right) \\
=W_{1}\left(f_{1}, \theta_{2}\right) \leq \int_{\bar{\Omega}^{3}}\left|x_{1}-z_{2}\right| \mathrm{d} \gamma_{f_{1}, 1,2}\left(x_{1}, z_{1}, z_{2}\right) .
\end{gathered}
$$

By triangular inequality and the continuity of the integrands, we get

$\left|x_{1}-z_{1}\right|+\left|z_{1}-z_{2}\right|=\left|x_{1}-z_{2}\right|$, i.e., $z_{1} \in\left[x_{1}, z_{2}\right]$ for all $\left(x_{1}, z_{1}, z_{2}\right) \in \operatorname{supp}\left(\gamma_{f_{1}, 1,2}\right)$.

In the same way,

$$
\left|z_{1}-z_{2}\right|+\left|z_{2}-x_{2}\right|=\left|z_{1}-x_{2}\right| \text {, i.e., } z_{2} \in\left[z_{1}, x_{2}\right] \text { for all }\left(z_{1}, z_{2}, x_{2}\right) \in \operatorname{supp}\left(\gamma_{1,2, f_{2}}\right) \text {. }
$$

If there exists $\left(z_{1}, z_{2}\right) \in \operatorname{supp}\left(\gamma_{1,2}\right)$ such that $z_{1} \neq z_{2}$ then, using (3.15) and (3.16), there are $x_{1} \in \operatorname{supp}\left(f_{1}\right), x_{2} \in \operatorname{supp}\left(f_{2}\right)$ such that $z_{1} \in\left[x_{1}, z_{2}\right], z_{2} \in\left[z_{1}, x_{2}\right]$ and therefore $z_{1}, z_{2} \in\left[x_{1}, x_{2}\right]$ (by $z_{1} \neq z_{2}$ ), a contradiction with the assumption (H). This shows that $\theta_{1}=\theta_{2}$. At last, using (3.11), we obtain $\nu=\Theta-\theta_{i} \leq \Theta, i=1,2$.

4. Uniqueness of optimal matching measure. This section concerns the proof for the uniqueness of optimal matching measure in Theorem 2.1. Let $\rho$ be an optimal matching measure. By Corollary 2.5 and Theorem 2.3, setting $\nu:=\Theta-\rho$, we have

$$
\left\{\begin{array}{l}
-\nabla \cdot \Phi_{i}=\Theta-\nu-f_{i} \text { in } \mathbb{R}^{N}, i=1,2 \\
\frac{\Phi_{i}}{\left|\Phi_{i}\right|}=\nabla_{\left|\Phi_{i}\right|} u_{i}\left|\Phi_{i}\right| \text {-a.e. in } \bar{\Omega}, i=1,2 \\
u_{1}+u_{2}=0 \quad \nu \text {-a.e. in } \bar{\Omega}
\end{array}\right.
$$


where $\Phi_{i}$ and $u_{i}$ are optimal flows and Kantorovich potentials, respectively. To prove the uniqueness of optimal matching measure under the assumption $(\mathrm{H})$, we establish precise expression of $\nu$ w.r.t. $\Theta$ and $u_{i}$, for $i=1,2$. More precisely, we have

Proposition 4.1. Assume that $\Theta \in L^{1}(\Omega)^{+}, f_{i} \in \mathcal{M}_{b}^{+}(\Omega) i=1,2$ and that $\left(\Phi_{1}, \Phi_{2}, \nu, u_{1}, u_{2}\right) \in \mathcal{M}_{b}(\bar{\Omega})^{N} \times \mathcal{M}_{b}(\bar{\Omega})^{N} \times \mathcal{M}_{b}^{+}(\bar{\Omega}) \times$ Lip $_{1}(\Omega) \times$ Lip $_{1}(\Omega)$ satisfies the $P D E$ (4.1). Under the assumption $(\mathrm{H})$, we have $\nu \leq \Theta$ and

$$
\nu=\Theta\left\llcorner\left[u_{1}+u_{2}=0\right],\right.
$$

where the set $\left[u_{1}+u_{2}=0\right]:=\left\{x \in \Omega: u_{1}(x)+u_{2}(x)=0\right\}$.

The proof of this result follows as a consequence of the following lemmas.

Lemma 4.2. Let $u, v$ be 1-Lipschitz functions on $\Omega$ such that $u+v \leq 0$ on $\Omega$. Assume that $u\left(y_{1}\right)-u\left(x_{1}\right)=\left|y_{1}-x_{1}\right|$ and that $u(z)+v(z)=0$ for some $z \in\left[x_{1}, y_{1}\right]$ the segment joining $x_{1}$ to $y_{1}$. Then

$$
u(s)+v(s)=0 \quad \forall s \in\left[z, y_{1}\right] .
$$

Moreover, if $x_{2} \in \Omega$ is such that $v\left(y_{1}\right)-v\left(x_{2}\right)=\left|y_{1}-x_{2}\right|$, then $z, y_{1}$ and $x_{2}$ are aligned.

Proof. We prove first that

$$
v(s)=v(z)-|s-z| \quad \forall s \in\left[z, y_{1}\right] .
$$

Since $u$ is 1-Lipschitz and $u\left(y_{1}\right)-u\left(x_{1}\right)=\left|y_{1}-x_{1}\right|$, we have

$$
u(s)=u(z)+|s-z| \quad \forall s \in\left[z, y_{1}\right] .
$$

Using the fact that $u+v \leq 0$, we have

$$
\begin{aligned}
v(s) & \leq-u(s) \\
& =-u(z)-|s-z| \\
& =v(z)-|s-z| \quad \forall s \in\left[z, y_{1}\right] .
\end{aligned}
$$

Since $v$ is 1-Lipschitz, we get the equality (4.3) and thus (4.2) (by $u(z)+v(z)=0$ and (4.4)).

At last, following (4.3) with $s=y_{1}$,

$$
v\left(y_{1}\right)=v(z)-\left|y_{1}-z\right|,
$$

we get, for $x_{2}$ as in the hypothesis,

$$
\begin{aligned}
\left|z-x_{2}\right| & \geq v(z)-v\left(x_{2}\right) \\
& =\left|z-y_{1}\right|+v\left(y_{1}\right)-v\left(x_{2}\right) \\
& =\left|z-y_{1}\right|+\left|y_{1}-x_{2}\right| .
\end{aligned}
$$

This implies that $z, y_{1}$ and $x_{2}$ are aligned.

Next, we need the following behaviors of $f_{i}$ and $\Phi_{i}, i=1,2$ on the set $\left[u_{1}+u_{2}=0\right]$.

Lemma 4.3. Assume that $f_{1}, f_{2}, \Theta \in \mathcal{M}_{b}^{+}(\Omega)$ and that $(\mathrm{H})$ holds. Suppose that $\left(\Phi_{1}, \Phi_{2}, \nu, u_{1}, u_{2}\right)$ satisfies the PDE (4.1). Then 
(i) $f_{1}\left\llcorner\left[u_{1}+u_{2}=0\right]=f_{2}\left\llcorner\left[u_{1}+u_{2}=0\right]=0\right.\right.$;

(ii) $\mathcal{L}^{N}\left(\operatorname{supp}\left(\Phi_{1}\right) \cap\left[u_{1}+u_{2}=0\right]\right)=\mathcal{L}^{N}\left(\operatorname{supp}\left(\Phi_{2}\right) \cap\left[u_{1}+u_{2}=0\right]\right)=0$, where $\mathcal{L}^{N}$ is the Lebesgue measure on $\mathbb{R}^{N}$.

Proof. (i) Thanks to Theorem 2.7, we have $\nu \leq \Theta$. Let us first show that $f_{1}\left\llcorner\left[u_{1}+u_{2}=0\right]=0\right.$. Assume on the contrary that the conclusion is not true. Then there exist $x_{1} \in\left[u_{1}+u_{2}=0\right]$ and $y_{1} \in \operatorname{supp}(\Theta-\nu)$ such that $\left(x_{1}, y_{1}\right) \in \operatorname{supp}\left(\gamma_{1}\right)$, where $\gamma_{1}$ is the optimal plan from $f_{1}$ to $\Theta-\nu$. Since $u_{1}$ is a Kantorovich potential for $W_{1}\left(f_{1}, \Theta-\nu\right)$ (as explained in the characterization PDE (3.4)), we get

$$
u_{1}\left(y_{1}\right)-u_{1}\left(x_{1}\right)=\left|x_{1}-y_{1}\right| .
$$

Similarly, since $y_{1} \in \operatorname{supp}(\Theta-\nu)$, there is $x_{2} \in \operatorname{supp}\left(f_{2}\right)$ such that $\left(x_{2}, y_{1}\right) \in \operatorname{supp}\left(\gamma_{2}\right)$ and

$$
u_{2}\left(y_{1}\right)-u_{2}\left(x_{2}\right)=\left|x_{2}-y_{1}\right| .
$$

Using Lemma 4.2, we deduce that $x_{1}, y_{1}, x_{2}$ are aligned which contradicts with $(\mathrm{H})$. In the same way, we get $f_{2}\left\llcorner\left[u_{1}+u_{2}=0\right]=0\right.$.

(ii) Now, we prove that

$$
\mathcal{L}^{N}\left(\operatorname{supp}\left(\Phi_{1}\right) \cap\left[u_{1}+u_{2}=0\right]\right)=0 .
$$

Thanks to [1, Corollary 6.1] or [2, Theorem 6.2], we know that the set $E$ of right endpoints of maximal transport rays w.r.t. the Kantorovich potential $u_{1}$ satisfies $\mathcal{L}^{N}(E)=0$. To prove $(4.5)$, it is enough to show that

$$
\operatorname{supp}\left(\Phi_{1}\right) \cap\left[u_{1}+u_{2}=0\right] \subset E .
$$

Assume on the contrary that there exists $z \in \operatorname{supp}\left(\Phi_{1}\right) \cap\left[u_{1}+u_{2}=0\right]$ such that $z \notin E$. Then there exists $\left(x_{1}, y_{1}\right) \in \operatorname{supp}\left(f_{1}\right) \times \operatorname{supp}(\Theta-\nu)$ such that $z \in\left[x_{1}, y_{1}[\right.$ and $u_{1}\left(y_{1}\right)=u_{1}\left(x_{1}\right)+\left|y_{1}-x_{1}\right|$. On the other hand, since $y_{1} \in \operatorname{supp}(\Theta-\nu)$, there exists $x_{2} \in \operatorname{supp}\left(f_{2}\right)$ such that

$$
u_{2}\left(y_{1}\right)-u_{2}\left(x_{2}\right)=\left|y_{1}-x_{2}\right| .
$$

Since $u_{1}(z)+u_{2}(z)=0$, using Lemma 4.2 , we deduce that $z, y_{1}$ and $x_{2}$ are on a straight line. Thus $x_{1}, y_{1}$ and $x_{2}$ are aligned (by $z \in\left[x_{1}, y_{1}[\right.$ ). This is again a contradiction with $(\mathrm{H})$.

Proof of Proposition 4.1. We use notations of the above lemmas. By Theorem 2.7, we have $\nu \leq \Theta$. Following directly from the PDE (4.1), we have

$$
-\nabla \cdot\left(\Phi_{1}+\Phi_{2}\right)=2(\Theta-\nu)-\left(f_{1}+f_{2}\right) .
$$

This implies that

the measure $2(\Theta-\nu)-\left(f_{1}+f_{2}\right)$ is concentrated on $\operatorname{supp}\left(\Phi_{1}+\Phi_{2}\right)$.

In particular, $2(\Theta-\nu)\left\llcorner\left[u_{1}+u_{2}=0\right]-\left(f_{1}+f_{2}\right)\left\llcorner\left[u_{1}+u_{2}=0\right]\right.\right.$ is concentrated on $\left[u_{1}+u_{2}=0\right] \cap \operatorname{supp}\left(\Phi_{1}+\Phi_{2}\right)$. Thanks to Lemma 4.3 (i), we deduce that $2(\Theta-$ $\nu)\left\llcorner\left[u_{1}+u_{2}=0\right]\right.$ is concentrated on $\left[u_{1}+u_{2}=0\right] \cap \operatorname{supp}\left(\Phi_{1}+\Phi_{2}\right)$. Since $(\Theta-\nu) \in L^{1}$, using Lemma 4.3 (ii) and the fact that $\operatorname{supp}\left(\Phi_{1}+\Phi_{2}\right) \subset \operatorname{supp}\left(\Phi_{1}\right) \cup \operatorname{supp}\left(\Phi_{2}\right)$, we get

$$
(\Theta-\nu)\left\llcorner\left[u_{1}+u_{2}=0\right]=0 .\right.
$$


Since $u_{1}+u_{2}=0 \quad \nu$-a.e. in $\bar{\Omega}$, we deduce that

$$
\nu=\Theta\left\llcorner\left[u_{1}+u_{2}=0\right] .\right.
$$

We can now conclude the proof of the uniqueness of optimal matching measures with the following arguments.

Proof of Theorem 2.1. We fix a maximizer $\left(u_{1}, u_{2}\right)$ of the maximization problem (2.5). Then if $\rho_{1}$ and $\rho_{2}$ are optimal matching measures then $\nu_{i}:=\Theta-\rho_{i}, i=1,2$ satisfies the PDE (4.1). Thanks to Proposition 4.1, we get

$$
\rho_{1}=\Theta-\nu_{1}=\Theta\left\llcorner\left[u_{1}+u_{2}<0\right]=\Theta-\nu_{2}=\rho_{2} .\right.
$$

Remark 4.4. Following from the proof, the unique optimal matching measure has the form

$$
\rho=\Theta\left\llcorner\left[u_{1}+u_{2}<0\right],\right.
$$

for any maximizer $\left(u_{1}, u_{2}\right)$ of the dual problem $(2.5)$.

5. Numerical analysis for the problem. The present section concerns on numerical aspects of the matching problem.

\subsection{Convergence of the discretization.}

Proof of Theorem 2.8. The optimality condition of (2.10) is (see for instance [12])

$$
-\Lambda_{h}^{*}\left(\Phi_{1, h}, \Phi_{2, h}, \nu_{h}\right)=\partial \mathcal{F}_{h}\left(u_{1, h}, u_{2, h}\right) \text { in } V_{h}^{*}
$$

(or equivalently, $\left.-\left\langle\left(\Phi_{1, h}, \Phi_{2, h}, \nu_{h}\right), \Lambda_{h}(u, v)\right\rangle=\mathcal{F}_{h}(u, v) \forall(u, v) \in V_{h}\right)$, and

$$
\left(\Phi_{1, h}, \Phi_{2, h}, \nu_{h}\right) \in \partial \mathcal{G}_{h}\left(\Lambda_{h}\left(u_{1, h}, u_{2, h}\right)\right) .
$$

Writing these in details, we have

$-\left\langle\Phi_{1, h}, \nabla u\right\rangle-\left\langle\Phi_{2, h}, \nabla v\right\rangle-\left\langle\nu_{h}, u+v\right\rangle=\left\langle f_{1, h}, u\right\rangle+\left\langle f_{2, h}, v\right\rangle-\left\langle\Theta_{h}, u+v\right\rangle \forall(u, v) \in V_{h}$,

and

$$
\left\{\begin{array}{l}
\Phi_{1, h} \in \partial \mathbb{I}_{B_{\left(Y_{h},\|\cdot\| \infty\right)}}\left(\nabla u_{1, h}\right) \\
\Phi_{2, h} \in \partial \mathbb{I}_{B_{\left(Y_{h},\|\cdot\| \infty\right)}\left(\nabla u_{2, h}\right)}\left(u_{1, h}+u_{2, h}\right) .
\end{array}\right.
$$

Choosing test functions $u \equiv 1, v \equiv 0$ in (5.1) and using the fact that $f_{1, h}(\Omega)<\Theta_{h}(\Omega)$, we have that $\nu_{h} \neq 0$ and $\left\{\nu_{h}\right\}$ is bounded in $L^{1}(\Omega)$. Since $\nu_{h} \in \partial \mathbb{I}_{\left\{z \in E_{h}: z \leq 0\right\}}\left(u_{1, h}+\right.$ $\left.u_{2, h}\right)$, we get $\nu_{h} \geq 0$ and $\left\langle u_{1, h}+u_{2, h}, \nu_{h}\right\rangle=0$. Since $\nu_{h} \neq 0$, there exists $x_{h} \in \bar{\Omega}$ such that $u_{1, h}\left(x_{h}\right)+u_{2, h}\left(x_{h}\right)=0$. Combining this with the fact $\int u_{1, h}=\int u_{2, h}$, we imply that $\left\{u_{1, h}\right\}$ and $\left\{u_{2, h}\right\}$ are bounded in $C(\bar{\Omega})$. Since $u_{1, h}, u_{2, h}$ are 1-Lipschitz functions, up to a subsequence (using the Ascoli-Arzela Theorem),

$$
u_{1, h} \rightrightarrows u_{1}^{*}, u_{2, h} \rightrightarrows u_{2}^{*} \text { uniformly on } \bar{\Omega} .
$$

It is clear that $u_{1}^{*}, u_{2}^{*}$ are 1-Lipschitz and $u_{1}^{*}+u_{2}^{*} \leq 0$ on $\bar{\Omega}$. 
On the other hand, using the optimality of $\left(u_{1, h}, u_{2, h}\right),\left(\Phi_{1, h}, \Phi_{2, h}, \nu_{h}\right)$ and the duality equality for (2.10), we have

$$
\mathcal{F}_{h}\left(u_{1, h}, u_{2, h}\right)+\mathcal{G}_{h}\left(\Lambda_{h}\left(u_{1, h}, u_{2, h}\right)\right)=-\mathcal{F}_{h}^{*}\left(-\Lambda_{h}^{*}\left(\Phi_{1, h}, \Phi_{2, h}, \nu_{h}\right)\right)-\mathcal{G}_{h}^{*}\left(\Phi_{1, h}, \Phi_{2, h}, \nu_{h}\right),
$$

or more explicitly,

$$
\begin{gathered}
\left\langle f_{1, h}, u_{1, h}\right\rangle+\left\langle f_{1, h}, u_{2, h}\right\rangle-\left\langle\Theta_{h}, u_{1, h}+u_{2, h}\right\rangle \\
=-\sup \left\{\left\langle\Phi_{1, h}, q\right\rangle: q \in Y_{h},|q(x)| \leq 1, \text { a.e. } x \in \Omega\right\} \\
-\sup \left\{\left\langle\Phi_{2, h}, q\right\rangle: q \in Y_{h},|q(x)| \leq 1 \text {, a.e. } x \in \Omega\right\} .
\end{gathered}
$$

Using the boundedness of $\left(u_{1, h}, u_{2, h}\right)$, we obtain that $\Phi_{1, h}$ and $\Phi_{2, h}$ are bounded in $L^{1}(\Omega)^{N}$. Thus, up to a subsequence,

$$
\left(\Phi_{1, h}, \Phi_{2, h}, \nu_{h}\right) \rightarrow\left(\Phi_{1}, \Phi_{2}, \nu\right) \text { weakly* in } \mathcal{M}_{b}(\bar{\Omega})^{N} \times \mathcal{M}_{b}(\bar{\Omega})^{N} \times \mathcal{M}_{b}(\bar{\Omega}) .
$$

Then $\left(\Phi_{1}, \Phi_{2}, \nu\right)$ is feasible for the problem (MMF). Indeed, thanks to (5.1) and the nonnegativity of $\nu_{h}$, we obtain that

$\left\langle\Phi_{1}, \nabla u\right\rangle+\left\langle\Phi_{2}, \nabla v\right\rangle+\langle\nu, u+v\rangle=-\left\langle f_{1}, u\right\rangle-\left\langle f_{2}, v\right\rangle+\langle\Theta, u+v\rangle \forall(u, v) \in V:=C^{1}(\bar{\Omega}) \times C^{1}(\bar{\Omega})$, and

$$
\nu \geq 0
$$

i.e., the feasibility of $\left(\Phi_{1}, \Phi_{2}, \nu\right)$. Now, we show the optimality of $\left(u_{1}^{*}, u_{2}^{*}\right)$ and $\left(\Phi_{1}, \Phi_{2}, \nu\right)$. Thanks to Theorem 2.3, it is sufficient to show that

$$
-\left\langle f_{1}, u_{1}^{*}\right\rangle-\left\langle f_{2}, u_{2}^{*}\right\rangle+\left\langle\Theta, u_{1}^{*}+u_{2}^{*}\right\rangle \geq\left|\Phi_{1}\right|(\bar{\Omega})+\left|\Phi_{2}\right|(\bar{\Omega}) .
$$

To this aim, let $q_{1}, q_{2} \in C(\bar{\Omega})^{N}$ be such that $\left|q_{1}(x)\right| \leq 1,\left|q_{2}(x)\right| \leq 1 \forall x \in \bar{\Omega}$ and $q_{1, h}, q_{2, h} \in Y_{h}$ be such that $\left\|q_{i, h}-q_{i}\right\|_{L^{\infty}(\Omega)} \rightarrow 0$ as $h \rightarrow 0, i=1,2$. By the fact that

$$
\left|q_{i, h}(x)\right|=\left|q_{i}(x)\right|+\left|q_{i, h}(x)\right|-\left|q_{i}(x)\right| \leq 1+O(h) \text { a.e. } x \in \Omega,
$$

and, taking $\frac{q_{i, h}}{1+O(h)}$ if necessary, we can assume that

$$
q_{i, h} \in Y_{h},\left|q_{i, h}(x)\right| \leq 1 \text { a.e. } x \in \Omega \text { and }\left\|q_{i, h}-q_{i}\right\|_{L^{\infty}(\Omega)} \rightarrow 0 \text { as } h \rightarrow 0, i=1,2 .
$$

We see that

$$
\begin{aligned}
\left\langle\Phi_{1}, q_{1}\right\rangle & =\left\langle\Phi_{1, h}, q_{1, h}\right\rangle+\left\langle\Phi_{1}-\Phi_{1, h}, q_{1}\right\rangle+\left\langle\Phi_{1, h}, q_{1}-q_{1, h}\right\rangle \\
& \leq \sup \left\{\left\langle\Phi_{1, h}, q\right\rangle: q \in Y_{h},|q(x)| \leq 1 \text { a.e. } x \in \Omega\right\}+O(h) .
\end{aligned}
$$

Similarly,

$$
\left\langle\Phi_{2}, q_{2}\right\rangle \leq \sup \left\{\left\langle\Phi_{2, h}, q\right\rangle: q \in Y_{h},|q(x)| \leq 1 \text { a.e. } x \in \Omega\right\}+O(h) .
$$

Combining these with (5.2),

$$
-\left\langle f_{1, h}, u_{1, h}\right\rangle-\left\langle f_{2, h}, u_{2, h}\right\rangle+\left\langle\Theta_{h}, u_{1, h}+u_{2, h}\right\rangle+O(h) \geq\left\langle\Phi_{1}, q_{1}\right\rangle+\left\langle\Phi_{2}, q_{2}\right\rangle .
$$

Letting $h \rightarrow 0$ and taking supremum in $q_{1}, q_{2}$, we get the desired inequality (5.3). 
5.2. Solving the discretized problem. Our task is now to solve the finitedimensional problem (2.10). We recall first the ALG2 method. Let $V$ and $Z$ be two Hilbert spaces. Let us consider the problem

$$
\inf _{u \in V} \mathcal{F}(u)+\mathcal{G}(\Lambda u)
$$

where $\mathcal{F}: V \longrightarrow(-\infty,+\infty]$ and $\mathcal{G}: Z \longrightarrow(-\infty,+\infty]$ are convex, l.s.c. and $\Lambda \in$ $\mathcal{L}(V, Z)$. The Fenchel-Rockafellar dual problem is

$$
\sup _{\sigma \in Z}\left(-\mathcal{F}^{*}\left(-\Lambda^{*} \sigma\right)-\mathcal{G}^{*}(\sigma)\right) .
$$

Introducing new variable $q \in Z$ to the primal problem (5.4), we rewrite it in the form

$$
\inf _{(u, q) \in V \times Z: \Lambda u=q} \mathcal{F}(u)+\mathcal{G}(q) .
$$

Consider the augmented Lagrangian w.r.t. the quadratic term

$$
L(u, q ; \sigma):=\mathcal{F}(u)+\mathcal{G}(q)+\langle\sigma, \Lambda u-q\rangle+\frac{r}{2}|\Lambda u-q|^{2}, r>0 .
$$

The ALG2 algorithm (see e.g. [10, 15]) is a primal-dual method (i.e., providing numerical solutions of both primal and dual problems) consisting of 3 steps. Known $q_{i}, \sigma_{i}$, the next step $\left(u_{i+1}, q_{i+1}, \sigma_{i+1}\right)$ is computed as follows: Fix any parameter $r>0$ (in practice $r=1,2$ ),

- Step 1: Minimizing $\inf _{u} L\left(u, q_{i} ; \sigma_{i}\right)$, i.e.

$$
u_{i+1}=\underset{u \in V}{\arg \min }\left\{\mathcal{F}(u)+\left\langle\sigma_{i}, \Lambda u\right\rangle+\frac{r}{2}\left|\Lambda u-q_{i}\right|^{2}\right\} .
$$

- Step 2: Minimizing $\inf _{q \in Z} L\left(u_{i+1}, q ; \sigma_{i}\right)$, i.e.

$$
q_{i+1}=\underset{q \in Z}{\arg \min }\left\{\mathcal{G}(q)-\left\langle\sigma_{i}, q\right\rangle+\frac{r}{2}\left|\Lambda u_{i+1}-q\right|^{2}\right\} .
$$

- Step 3: Update the multiplier $\sigma$,

$$
\sigma_{i+1}=\sigma_{i}+r\left(\Lambda u_{i+1}-q_{i+1}\right) .
$$

Now, apply the ALG2 to our discretized problem (2.10). To simplify the notations, let us drop out the subscript $h$ in $u_{1, h}, u_{2, h}, \Phi_{1, h}, \Phi_{2, h}, \nu_{h}$. We denote by $\Phi_{1}^{i}, \Phi_{2}^{i}, \nu^{i}, u_{1}^{i}, u_{2}^{i}, p^{i}, q^{i}, s^{i}$ the values at iteration $i$. Known $\left(p^{i}, q^{i}, s^{i}\right),\left(\Phi_{1}^{i}, \Phi_{2}^{i}, \nu^{i}\right)$,

- Step 1:

$$
\begin{gathered}
\left(u_{1}^{i+1}, u_{2}^{i+1}\right)=\underset{\left(u_{1}, u_{2}\right) \in V_{h}}{\arg \min } \mathcal{F}_{h}\left(u_{1}, u_{2}\right)+\left\langle\left(\Phi_{1}^{i}, \Phi_{2}^{i}, \nu^{i}\right), \Lambda_{h}\left(u_{1}, u_{2}\right)\right\rangle+\frac{r}{2}\left|\Lambda_{h}\left(u_{1}, u_{2}\right)-\left(p^{i}, q^{i}, s^{i}\right)\right|^{2} \\
=\underset{\left(u_{1}, u_{2}\right) \in V_{h}}{\arg \min }\left\langle u_{1}, f_{1, h}\right\rangle+\left\langle u_{2}, f_{2, h}\right\rangle-\left\langle u_{1}+u_{2}, \Theta_{h}\right\rangle+\left\langle\Phi_{1}^{i}, \nabla u_{1}\right\rangle+\left\langle\Phi_{2}^{i}, \nabla u_{2}\right\rangle \\
+\left\langle\nu^{i}, u_{1}+u_{2}\right\rangle+\frac{r}{2}\left|\nabla u_{1}-p^{i}\right|^{2}+\frac{r}{2}\left|\nabla u_{2}-q^{i}\right|^{2}+\frac{r}{2}\left|u_{1}+u_{2}-s^{i}\right|^{2} .
\end{gathered}
$$

- Step 2:

$$
\begin{gathered}
\left(p^{i+1}, q^{i+1}, s^{i+1}\right)=\underset{(p, q, s) \in Z_{h}}{\arg \min } \mathcal{G}_{h}(p, q, s)-\left\langle\left(\Phi_{1}^{i}, \Phi_{2}^{i}, \nu^{i}\right),(p, q, s)\right\rangle+\frac{r}{2}\left|\Lambda_{h}\left(u_{1}^{i+1}, u_{2}^{i+1}\right)-(p, q, s)\right|^{2} \\
=\underset{(p, q, s) \in Z_{h}}{\arg \min } \mathbb{I}_{\left(Y_{h},\|\cdot\| \infty\right)}(p)+\mathbb{I}_{B_{\left(Y_{h},\|\cdot\| \infty\right)}}(q)+\mathbb{I}_{\left\{s \in E_{h}: s \leq 0\right\}}(s)-\left\langle\Phi_{1}^{i}, p\right\rangle-\left\langle\Phi_{2}^{i}, q\right\rangle-\left\langle\nu^{i}, s\right\rangle \\
\quad+\frac{r}{2}\left|\nabla u_{1}^{i+1}-p\right|^{2}+\frac{r}{2}\left|\nabla u_{2}^{i+1}-q\right|^{2}+\frac{r}{2}\left|u_{1}^{i+1}+u_{2}^{i+1}-s\right|^{2} .
\end{gathered}
$$


- $\quad$ Step 3:

$\left(\Phi_{1}^{i+1}, \Phi_{2}^{i+1}, \nu^{i+1}\right)=\left(\Phi_{1}^{i}, \Phi_{2}^{i}, \nu^{i}\right)+r\left(\nabla u_{1}^{i+1}-p^{i+1}, \nabla u_{2}^{i+1}-q^{i+1}, u_{1}^{i+1}+u_{2}^{i+1}-s^{i+1}\right)$.

Let us give more details of the above iteration. Overall, Step 1 is a quadratic programming. Step 2 can be calculated explicitly and Step 3 updates obviously. We denote by $\operatorname{Proj}_{C}($.$) the projection onto a closed convex subset C$.

- In Step 1: We split variables $u_{1}$ and $u_{2}$, i.e., first minimizing w.r.t. $u_{1}$ and using $u_{1}^{i+1}$ to calculate $u_{2}^{i+1}$.

1. For $u_{1}^{i+1}$,

$$
u_{1}^{i+1}=\underset{u \in E_{h}}{\arg \min }\left\langle u, f_{1, h}-\Theta_{h}\right\rangle+\left\langle\Phi_{1}^{i}, \nabla u\right\rangle+\left\langle\nu^{i}, u\right\rangle+\frac{r}{2}\left|\nabla u-p^{i}\right|^{2}+\frac{r}{2}\left|u+u_{2}^{i}-s^{i}\right|^{2} .
$$

This is a quadratic problem with the associated linear equation:

$r\left\langle\nabla u_{1}^{i+1}, \nabla \phi\right\rangle+r\left\langle u_{1}^{i+1}, \phi\right\rangle=\left\langle\Theta_{h}-f_{1, h}-\nu^{i}, \phi\right\rangle+\left\langle r p^{i}-\Phi_{1}^{i}, \nabla \phi\right\rangle+r\left\langle s^{i}-u_{2}^{i}, \phi\right\rangle \forall \phi \in E_{h}$.

2. Similarly for $u_{2}^{i+1}$,

$r\left\langle\nabla u_{2}^{i+1}, \nabla \phi\right\rangle+r\left\langle u_{2}^{i+1}, \phi\right\rangle=\left\langle\Theta_{h}-f_{2, h}-\nu^{i}, \phi\right\rangle+\left\langle r q^{i}-\Phi_{2}^{i}, \nabla \phi\right\rangle+r\left\langle s^{i}-u_{1}^{i+1}, \phi\right\rangle \forall \phi \in E_{h}$.

- In Step 2: Since the function $\mathcal{G}(p, q, s)$ has the form of $\mathcal{G}_{1}(p)+\mathcal{G}_{2}(q)+\mathcal{G}_{3}(s)$, we solve them separately.

1. For $s^{i+1}$, if we choose $P_{2}$ finite element for $s^{i+1}$,

$$
\begin{aligned}
s^{i+1} & =\underset{s \in P_{2}}{\arg \min }\left\{\mathbb{I}_{[s \leq 0]}-\left\langle\nu^{i}, s\right\rangle+\frac{r}{2}\left|u_{1}^{i+1}+u_{2}^{i+1}-s\right|^{2}\right\} \\
& =\operatorname{Proj}_{\left\{s \in P_{2}: s \leq 0\right\}}\left(u_{1}^{i+1}+u_{2}^{i+1}+\frac{\nu^{i}}{r}\right) .
\end{aligned}
$$

This is computed in pointwise, i.e., at vertices $x_{k}$ of a given grid,

$$
s^{i+1}\left(x_{k}\right)=\operatorname{Proj}_{[r \in \mathbb{R}: r \leq 0]}\left(u_{1}^{i+1}\left(x_{k}\right)+u_{2}^{i+1}\left(x_{k}\right)+\frac{\nu^{i}\left(x_{k}\right)}{r}\right) .
$$

2. For $p^{i+1}$ and $q^{i+1}$, similarly, at each vertice $x_{l}$,

$$
p^{i+1}\left(x_{l}\right)=\operatorname{Proj}_{\bar{B}(0,1)}\left(\nabla u_{1}^{i+1}\left(x_{l}\right)+\frac{\Phi_{1}^{i}\left(x_{l}\right)}{r}\right)
$$

and

$$
q^{i+1}\left(x_{l}\right)=\operatorname{Proj}_{\bar{B}(0,1)}\left(\nabla u_{2}^{i+1}\left(x_{l}\right)+\frac{\Phi_{2}^{i}\left(x_{l}\right)}{r}\right) .
$$

6. Numerical experiments. We base on $[4,5,17]$ and on FreeFem $++[16]$ to give some numerical examples. We use $P_{2}$ finite element for $u_{1}^{i}, u_{2}^{i}, s^{i}, \nu^{i}$ and $P_{1}$ finite element for $\Phi_{1}^{i}, \Phi_{2}^{i}, p^{i}, q^{i}$.

6.1. Stopping criterion. The measures $f_{1}, f_{2}$ and $\Theta$ are approximated by nonnegative regular functions that we denote again by $f_{1}, f_{2}$ and $\Theta$. We use the PDE of optimality condition as stopping criteria: 
1. $\mathrm{MIN}:=\min \left\{\min _{\bar{\Omega}}\left(-u_{1}(x)-u_{2}(x)\right), \min _{\bar{\Omega}} \nu(x)\right\}$.

2. Lip $:=\max \left\{\max _{\bar{\Omega}}\left|\nabla u_{1}(x)\right|, \max _{\bar{\Omega}}\left|\nabla u_{2}(x)\right|\right\}$.

3. DIV $:=\frac{D i v_{1}+D_{i v_{2}}}{2}$, where

$$
\operatorname{Div}_{1}:=\left\|\nabla \cdot \Phi_{1}+\Theta-\nu-f_{1}\right\|_{L^{2}}, \quad \operatorname{Div}_{2}:=\left\|\nabla \cdot \Phi_{2}+\Theta-\nu-f_{2}\right\|_{L^{2}} .
$$

4. DUAL $:=\frac{\text { Dual }_{1}+\text { Dual }_{2}}{2}$, with

$$
\text { Dual }_{1}:=||\left|\Phi_{1}(x)\right|-\Phi_{1}(x) \cdot \nabla u_{1} \|_{L^{2}}, \text { Dual }_{2}:=\left\|\left|\Phi_{2}(x)\right|-\Phi_{2}(x) \cdot \nabla u_{2}\right\|_{L^{2}} .
$$

We expect that MIN $\geq 0$, Lip $\leq 1 ;$ DIV and DUAL are small.

6.2. Some examples. In all the examples below, we take $\Omega=[0,1] \times[0,1]$ and work on a grid $60 \times 60$. Computation time for each example is about 8 minutes on a PC Mac OSX 10.9.

Example 6.1. We take

$$
\begin{gathered}
f_{1}=4 \chi_{\left[(x-0.2)^{2}+(y-0.8)^{2}<0.01\right]}, \\
f_{2}=2 \chi_{\left[(x-0.8)^{2}+(y-0.8)^{2}<0.01\right]}+2 \chi_{\left[(x-0.2)^{2}+(y-0.2)^{2}<0.01\right]}, \\
\Theta=4 \chi_{\left[(x-0.5)^{2}+(y-0.5)^{2}<0.04\right]} .
\end{gathered}
$$

The optimal matching measure and optimal flows are given in Figure 3. Stopping criterion is given in Figure 4.

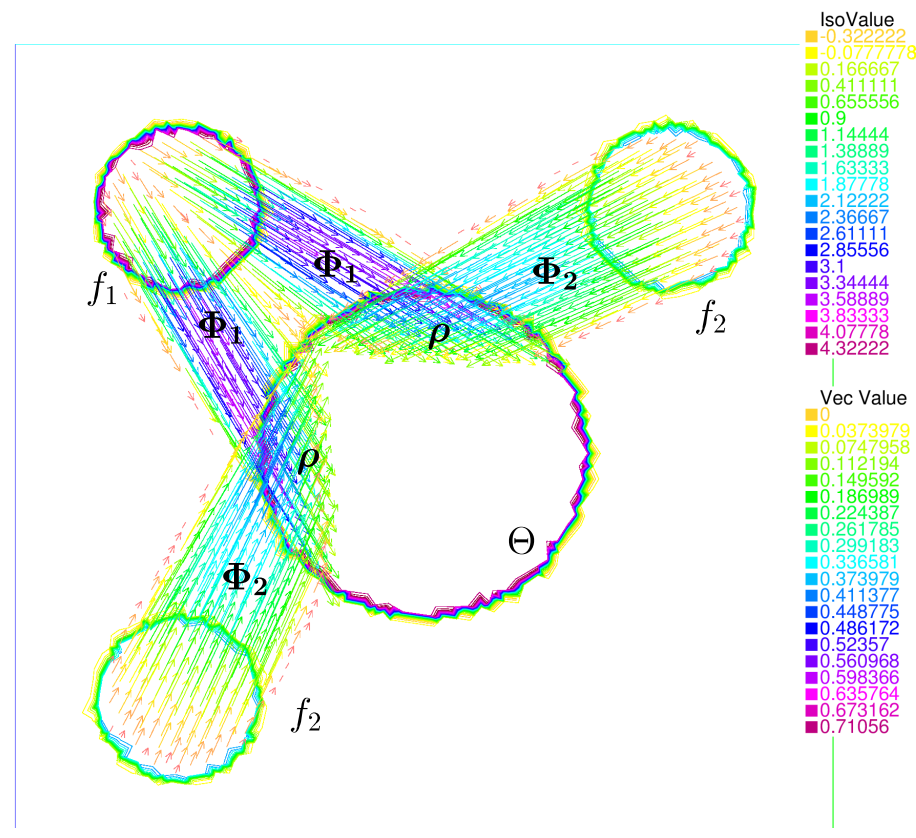

Fig. 3: Optimal matching measure and optimal flows 


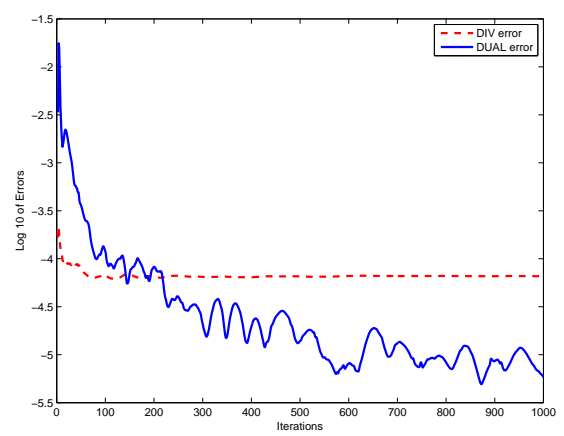

(a) $\log _{10}$ of DIV and DUAL errors

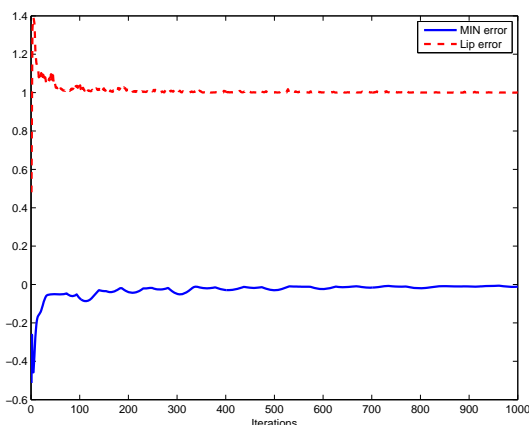

(b) MIN and Lip errors

Fig. 4: Stopping criterion

Example 6.2. The results are given in Figures 5 and 6 for

$$
\begin{gathered}
f_{1}=2 \chi_{\left[(x-0.2)^{2}+(y-0.8)^{2}<0.01\right]}+2 \chi_{\left[(x-0.8)^{2}+(y-0.2)^{2}<0.01\right]}, \\
f_{2}=2 \chi_{\left[(x-0.8)^{2}+(y-0.8)^{2}<0.01\right]}+2 \chi_{\left[(x-0.2)^{2}+(y-0.2)^{2}<0.01\right]}, \\
\Theta=4 \chi_{\left[(x-0.5)^{2}+(y-0.5)^{2}<0.04\right]} .
\end{gathered}
$$

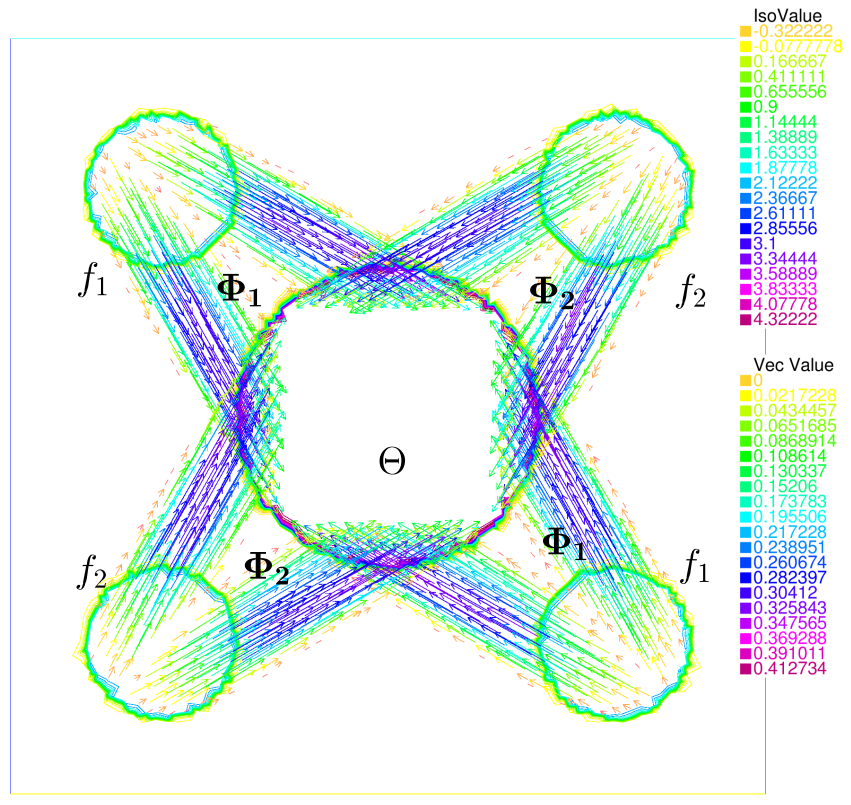

Fig. 5: Optimal matching measure and optimal flows 


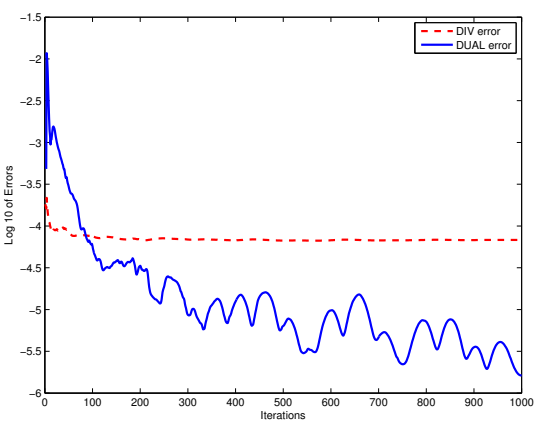

(a) $\log _{10}$ of DIV and DUAL errors

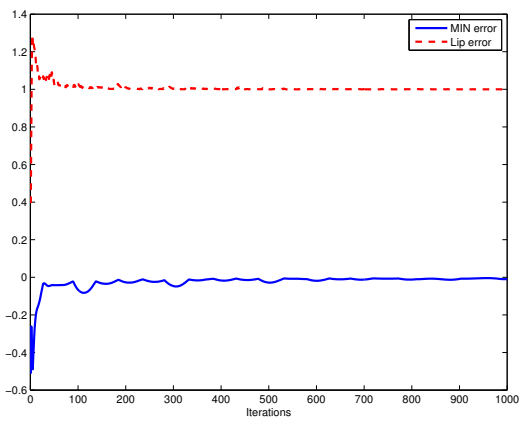

(b) MIN and Lip errors

Fig. 6: Stopping criterion

Example 6.3. We take

$$
\begin{gathered}
f_{1}=4 \chi_{\left[(x-0.1)^{2}+(y-0.9)^{2}<0.01\right]}, \\
f_{2}=4 \chi_{\left[(x-0.7)^{2}+(y-0.3)^{2}<0.01\right]}, \\
\Theta=4 \chi_{\left[(x-0.2)^{2}+(y-0.2)^{2}<0.04\right]}+4 \chi_{\left[(x-0.6)^{2}+(y-0.6)^{2}<0.0064\right]} .
\end{gathered}
$$

The results are given in Figures 7 and 8 .

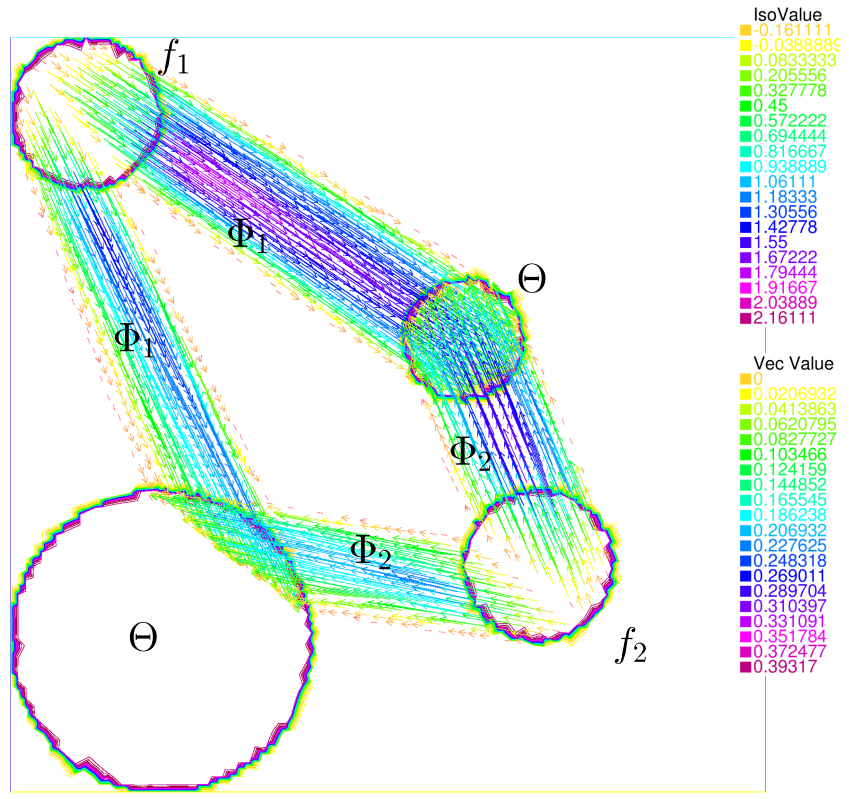

Fig. 7: Optimal matching measure and optimal flows 


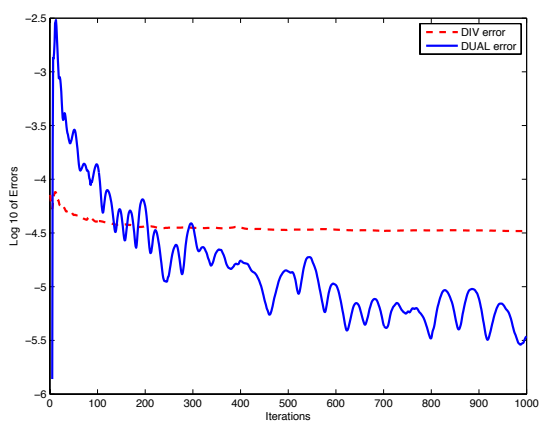

(a) $\log _{10}$ of DIV and DUAL errors

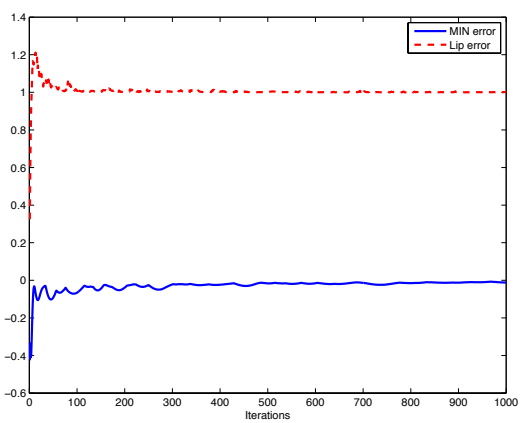

(b) MIN and Lip errors

Fig. 8: Stopping criterion

Acknowledgments. Part of this work has been done during a visit of J. Toledo to the Univ. of Limoges and a visit of N. Igbida and V. T. Nguyen to the Univ. of València, they want to acknowledge the warm hospitality found at these institutions. The authors would like to thank the anonymous referee for his careful reading of the paper and for his interesting suggestion of the idea of Lemma 3.2 that allows to simplify our first proof of Theorem 2.7.

\section{REFERENCES}

[1] L. Ambrosio, Lecture notes on optimal transport problems, Lecture Notes in Math., 1812 (2003), pp. 1-52.

[2] L. Ambrosio And A. Pratelli, Existence and stability results in the $L^{1}$ theory of optimal transportation, Lecture Notes in Math., 1813 (2003), pp. 123-160.

[3] J. W. Barrett and L. Prigozhin, Partial L ${ }^{1}$ Monge-Kantorovich problem: Variational formulation and numerical approximation, Interfaces Free Bound., 11 (2009), pp. 201-238.

[4] J. Benamou And Y. Brenier, A computational fluid mechanics solution to the MongeKantorovich mass transfer problem, Numer. Math., 84 (2000), pp. 375-393.

[5] J. D. Benamou And G. CARLier, Augmented Lagrangian methods for transport optimization, mean field games and degenerate elliptic equations, J. Optim. Theory Appl., 167 (2015), pp. $1-26$.

[6] G. Bouchitté, G. Buttazzo, and P. Seppercher, Energies with respect to a measure and applications to low dimensional structures, Calc. Var. Partial Differential Equations, 5 (1997), pp. 37-54.

[7] G. CARLIER, Duality and existence for a class of mass transportation problems and economic applications, Advances in Mathematical Economics, 5 (2003), pp. 1-21.

[8] G. Carlier and I. Ekeland, Matching for teams, Econom. Theory, 42 (2010), pp. 397-418.

[9] P. A. Chiappori, R. J. McCann, and L. P. Nesheim, Hedonic price equilibria, stable matching, and optimal transport: equivalence, topology, and uniqueness, Econom. Theory, 42 (2010), pp. 317-354.

[10] J. Eckstein And D. P. BertseKas, On the Douglas-Rachford splitting method and the proximal point algorithm for maximal monotone operators, Math. Program., 55 (1992), pp. 293318.

[11] I. Ekeland, An optimal matching problem, ESAIM Control Optim. Calc. Var., 11 (2005), pp. $57-71$.

[12] I. Ekeland And R. Teman, Convex analysis and variational problems, North-Holland American Elsevier, 1976.

[13] L. C. Evans and W. Gangbo, Differential equations methods for the Monge-Kantorovich mass transfer problem, Mem. Amer. Math. Soc., 137 (1999). 
[14] A. Figalli., The optimal partial transport problem, Arch. Ration. Mech. Anal., 195 (2010), pp. 533-560.

[15] R. Glowinski And P. L. Tallec, Augmented Lagrangian and operator-splitting methods in nonlinear mechanics, vol. 9, SIAM, 1989.

[16] F. Неснt, New development in Freefem++, J. Numer. Math., 20 (2012), pp. 251-266.

[17] N. Igbida And V. T. NGuYen, Augmented Lagrangian method for optimal partial transportation, IMA J. Numer. Anal., (2017), https://doi.org/10.1093/imanum/drw077.

[18] N. IgBida And V. T. NGuyen, Optimal partial mass transportation and obstacle MongeKantorovich equation, Submitted for publication, (2017).

[19] J. M. Mazón, J. D. Rossi, And J. Toledo, An optimal matching problem for the Euclidean distance, SIAM J. Math. Anal., 46 (2014), pp. 233-255.

[20] J. M. MAzón, J. D. Rossi, AND J. TOLEdo, An optimal matching problem with constraints, Submitted for publication, (2017).

[21] C. Villani, Optimal Transport, Old and New, vol. 338, Springer-Verlag, Berlin Heidelberg, 2009. 